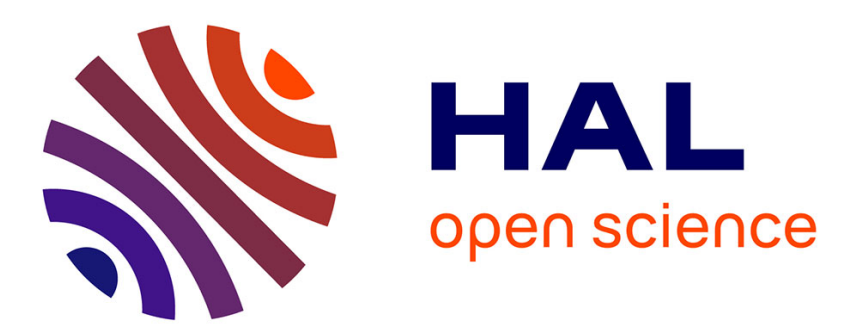

\title{
Various aspects of the radial and tangential energy dissipation in heavy ion collisions at bombarding energies in the range 4-12 $\mathrm{MeV} / \mathrm{A}$
}

\author{
M. Lefort
}

\section{- To cite this version:}

M. Lefort. Various aspects of the radial and tangential energy dissipation in heavy ion collisions at bombarding energies in the range 4-12 MeV/A. Heavy Ion Interactions At High Energies, Mar 1979, Erice, Italy. pp.197-240, 10.1016/0146-6410(80)90007-1 。 in2p3-00009030

HAL Id: in2p3-00009030

https://hal.in2p3.fr/in2p3-00009030

Submitted on 23 Jun 2020

HAL is a multi-disciplinary open access archive for the deposit and dissemination of scientific research documents, whether they are published or not. The documents may come from teaching and research institutions in France or abroad, or from public or private research centers.
L'archive ouverte pluridisciplinaire HAL, est destinée au dépôt et à la diffusion de documents scientifiques de niveau recherche, publiés ou non, émanant des établissements d'enseignement et de recherche français ou étrangers, des laboratoires publics ou privés. 


\title{
Various Aspects of the Radial and Tangential Energy Dissipation in Heavy Ion Collisions at Bombarding Energies in the Range 4 - 12 MeV.A.
}

\author{
MARC LEFORT \\ Institut de Physique Nucléaire 91406 ORSAY and \\ GANIL BP 5027 - 14021 CAEN, France
}

\section{INTRODUCTION AND SUMMARY.-}

The main purpose in this approach of the field of Deep Inelastic Collisions, is to focus our interest on perhaps the most striking feature of this class of Heavy Ion induced reactions, i.e., the very large energy loss observed in the relative motion, particularly drastic at large incident energies. It is indeed so prominent that it has led to the labelling as "deep inelastic" or "dissipative" process.

Since many review articles $\left({ }^{1-8}\right)$ have been made on the topics of D.I.R., it seems not necessary to repeat here all the well known characteristics. Nevertheless the study of the energy dissipation is particularly interesting for two reasons.

First, it should reveal which are the basic processes involved at the various stages of the damping - microscopic particle - hole excitations and nucleon exchange or collective dissipation modes like surface vibrations or dynamical deformations coupled to intrinsic degrees of freedom. One of the advantage of heavy ion reactions on nuclear fission is that the collision process itself furnishes a time scale along which one can follow the various steps of the energy relaxation. Therefore, dissipative phenomena in systems which initially are far from equilibrium can be studied as a function of time. Assuming that the system rotates with angular velocity

$$
\omega=l_{i} \hbar / y
$$

where $l_{i} \hbar$ is the entrance channel orbital angular momentum and $\mathcal{Y}$ the moment of inertia of the two colliding nuclei, the relationship between the relaxation time $\tau_{E}$ and the energy loss $\Delta E$ may be formulated

$$
\frac{\Delta E_{g}}{\Delta E}=\exp \left(\frac{\theta_{g}-\theta}{\omega \tau_{E}}\right)
$$

if $\Delta E$ is the energy loss at the grazing angle, $\theta$ and $\Delta E$ the energy loss at the obserfation angle, $\theta$, assuming an exponential $g$ decay of the energy peak as a function of time. 
The mechanism of the energy damping is still under discussion. It is generally admitted that the coupling which is exerted between collective "slow" degrees of freedom and intrinsic fast degrees of freedom is responsible for energy transfer. The energy flows from the collective motion to the intrinsic degrees because of the large phase space available. However transport theories using statistical assumptions are not expected to apply at the very beginning of the collision, for contact times shorter than the relaxation time of the intrinsic degree (for example $10^{-22} \mathrm{~s}$ for particle-hole excitation). There is certainly a fast dissipation which occurs in the approach phase of the collision where statistical assumptions are not yet valid. This is why results on rather low kinetic energy losses, i.e. on that part of the kinetic energy spectra which is close to the quasi-elastic peak present a great interest.

There are not yet enough evidence for making a clear cut choice between the absorption of energy by the excitation of high-lying surface modes (giant resonances) and the dissipation through Gross'piston model (wall formula) - i.e. when each single particle of the nuclear Fermi gas of one nucleus collides only once with the moving average potential wall of the collision partner, so that a macroscopic friction force results since many particles collide with the wall in a short time.

The second interesting point is to know where is found the missing kinetic energy. For moderate bombarding energies, most of the kinetic energy loss goes into excitation energy of the two fragments. Moreover they exhibit an isothermal sharing of the excitation energy at least in a number of experimental $\operatorname{data}(9-12)$.

After a very short historical survey, we shall present in section 2 typical experimental results showing in a number of systems energy spectra extending from the quasi-elastic small excitations up to very large excitation energies corresponding to a complete relaxation. Then we shall discuss the signification of the value of the kinetic energy at full relaxation in terms of Coulomb repulsion and rotational energy. Also we shall show that the energy distribution around the mean value is large and that statistical fluctuations are very important.

Also, in deep inelastic reactions not only the kinetic energy but the orbital angular momentum are partially transferred into the two fragments. There is obviousiy a correlation between the total kinetic energy loss and angular momentum transfer and this will be examined in section 4 , in connection with transport theories.

Between quasi-elastic scattering and complete relaxation there is a continuous spectrum of kinetic energy loss. It is quite obvious that the study of these partially damped phenomena may bring some knowledge on the dissipative mechanism. Section 5 will deal with such studies.

In section 6 , we shall review the main experimental evidences that the major part of the kinetic energy loss ends up into intrinsic excitation energy of the two fragments proportional to their mass. But al so some fraction of light particle emission exhibits a peculiar behaviour which may reflect some aspects of the mechanism of energy dissipation.

\section{A SURVEY OF TYPICAL EXPERIMENTAL RESULTS ON TOTAL KINETIC ENERGY LOSS.}

In the very first paper which was devoted to what is called now deep inelastic reactions, in 1961 , Kaufmann and Wolfgang $\left({ }^{13}\right)$ did not observe the kinetic energy of the fragments, because of the experimental technique they used, namely the collection of the reaction products and radioactivity measurements ; but they 
noticed the angular distribution which did not fit the expected quasi-elastic scattering behaviour, and they tried a theoretical interpretation in terms of "grazing collisions" which is still at the origin of the main ideas developed today. The first experimental discovery that two touching nuclei can lose most of
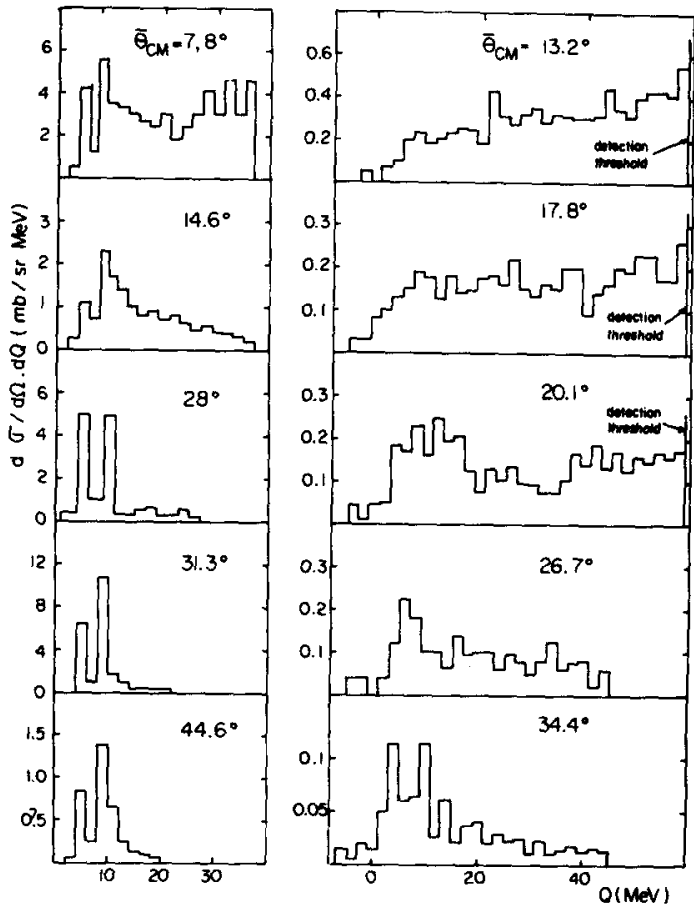

Fig. 1 : Energy balance distributions. On the left for ${ }^{13} \mathrm{C}$ products from the "incomplete fusion" reaction ${ }^{12} \mathrm{C}+\mathrm{Ag}$ at $86 \mathrm{MeV}$. on the right for ${ }^{16} 0$ products from the reaction ${ }^{14} \mathrm{~N}+\mathrm{Ag}$ at $113 \mathrm{MeV}$. (From Galin et al. 1970, ref. 15). Quasi-elastic events correspond to small $Q$ values. Dissipative events extend until the experimental detection threshold. their kinetic energy although they mainly keep their identity, was made in ORSAY around 1969. Amongst the products of the reaction ${ }^{14} \mathrm{~N}+\mathrm{Ag}$, it was shown $\left({ }^{15}\right)$ that the unexpected forward peaking component observed by Kaufmann and Wolfgang was corresponding to $10 \mathrm{~W}$ kinetic energies of the fragments. For the first time, a distinction was made between on one side quasi-elastic reactions characterized by a maximum in $\mathrm{d} \sigma / \mathrm{d} \Omega$ at the grazing angle and, on the other side, what was called incomplete fusion with a forward peaking anda Targe energy dissipation ( ${ }^{15}$ ). An illustration from the early work of 1970 is shown in figure 1 . In the same period, evidence for what was called "intermediate mechanism in interactions between complex nuclei" was published in Dubna by Volkov's group ${ }^{16}$ ). Energy spectra were obtained for the light fragments produced in multinucleon transfers by ${ }^{16} 0$ on ${ }^{27} \mathrm{Al},{ }^{51} \mathrm{~V}$ and ${ }^{93} \mathrm{Nb}$. Later on, a serie of well known papers appeared $\left({ }^{17}\right)$ on ${ }^{40} \mathrm{Ar}+232 \mathrm{Th}$ and the Wi lczynski's presentation ( $\left.{ }^{18}\right)$ of contour plots of $\frac{d 2 \sigma}{d E d} \theta$ versus $E$ and $\theta$ is so popular that we don't feel necessary to show it once more. "Wilczynski's thumbs" have appeared in droves for various systems including a recent picture obtained( ${ }^{19}$ ) in Lanchow-China in a rather modest study of ${ }^{12} \mathrm{C}$ on ${ }^{27} \mathrm{Al}$. Even 10 years after our first work done in ORSAY the curves shown in figure 2 exhibit a very good example of energy
dissipation, in this time where any heavy ion beam should transport masses heavier than 80 in order to be taken seriously into consideration. On this picture, one can see very nicely the continuous energy loss from a quasi-elastic discrete spectrum down to a very low kinetic energy for the ${ }^{1} i_{B}$ fragment.

As a general behaviour, energy spectra present a long descent from the quasi-elastic scattering energy followed by a broad peak centred at much lower energy. The distinction between the two parts is rather well marked when the detector is put forwards the grazing angle. However, close to the grazing angle, there This is clearly shown in figure 3 . Nevertheless, energy spectra suming the events corresponding to a 11
kind of fragments are certainly not the right thing to look at for a better un$\mathrm{d}$ rstanding of the energy dissipation. Measurements have been made for well defined
$Z$ values. 


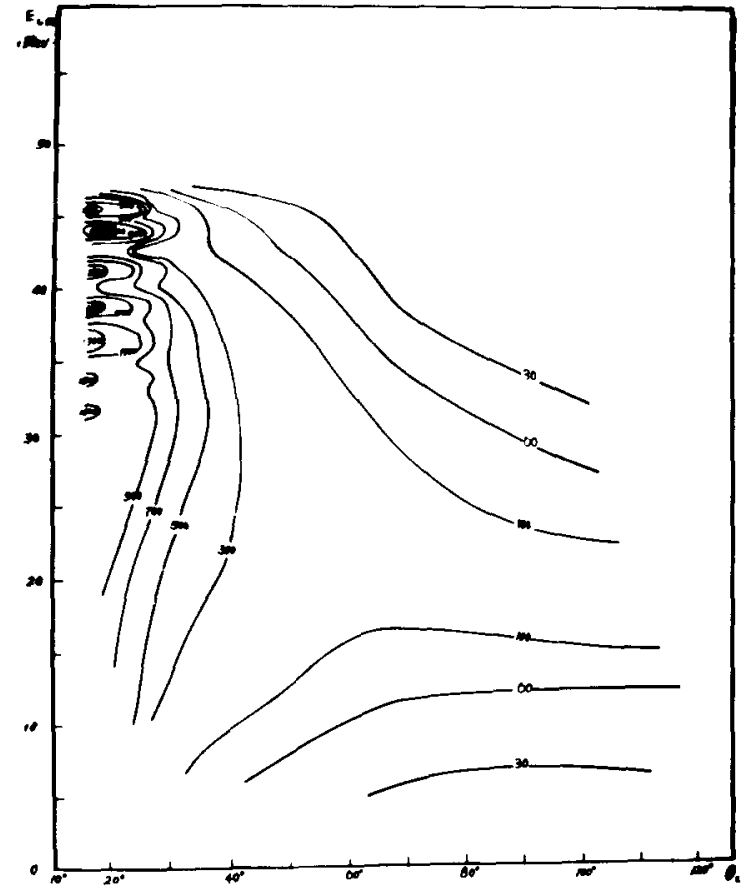

Fig. 2 : Contour lines for deep inelastic events versus total kinetic energy and angle of emission for the fragment ${ }^{10} \mathrm{~B}$ produced in the reaction $\left({ }^{12} \mathrm{C}+{ }^{27} \mathrm{Al}\right)$ with the $90 \mathrm{MeV}$ carbon beam delivered at the Institute of Modern Physics at Lanchow (People Republic of China) (ref. 19).

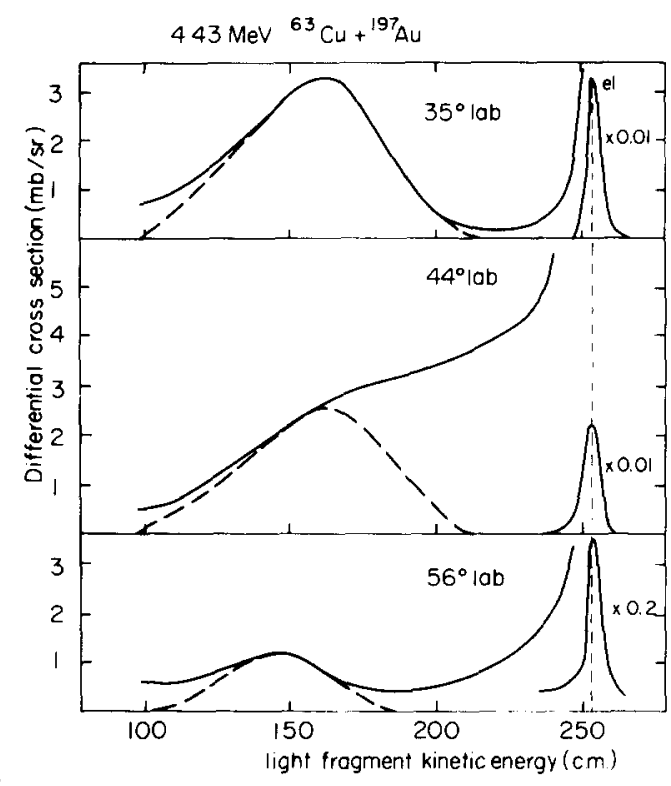

Fig. 3 : Partial and complete damping. Energy spectra for projectile-like frag. ments emitted at various angle in the reaction ${ }^{63} \mathrm{Cu}+19{ }^{7} \mathrm{Au}$ (ref. 23).

Furthermore it is important to consider each mass for a given $Z$, because it is not the same sort of isotopes which contribute to the low energy peak and to the region near by the quasi-elastic scattering. This is illustrated $\left.{ }^{20}\right)$ by figure 4 relative to Chlorine isotopes emitted in the reaction ${ }^{58} \mathrm{Ni}\left({ }^{40} \mathrm{Ar}, \mathrm{Cl}\right) \mathrm{Cu}$ at $E_{\text {lab }}=280 \mathrm{MeV}$. For ${ }^{39} \mathrm{Cl}$, due to the removal of a single proton, the only contribution appears at the grazing angle with a kinetic energy close to the elastic scattering value. There is no contribution to a low energy peak neither inside the grazing nor outside, because the nucleon interaction is deeper and leads to more than a single proton transfer. In contrast, ${ }^{34} \mathrm{Cl}$ exhibits no contribution from a quasi-elastic peak since there is already the loss of 6 nucleons. The spectrum of ${ }^{96} \mathrm{Cl}$ shows an intermediate behaviour with two broad peaks when the observation is made at an angle $\theta$ close to the grazing angle. 

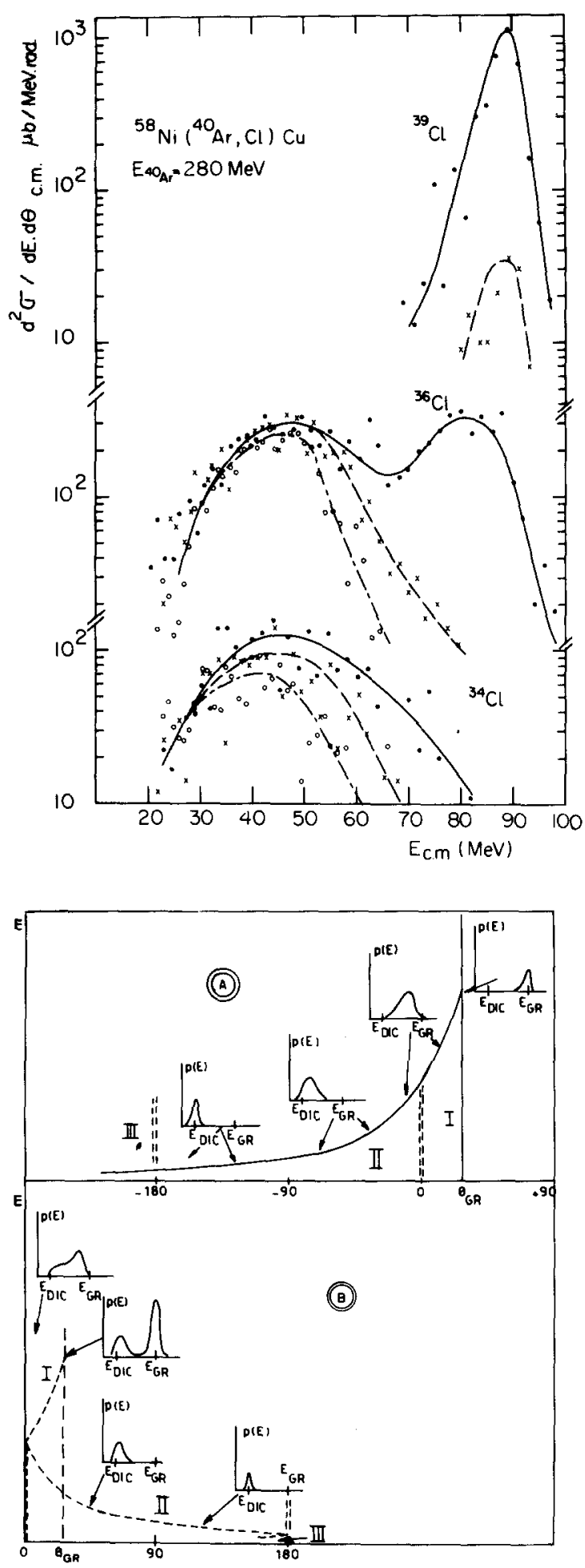

Fig. 4: Energy spectrum of chlorine isotopes emitted in the reaction ${ }^{58} \mathrm{Ni}\left({ }^{40} \mathrm{Ar}, \mathrm{Cl}\right) \mathrm{Cu}$ at $\mathrm{E}_{1 \mathrm{ab}}=280 \mathrm{MeV}$ (ref. 20) $\theta=18^{\circ}$ (dots), $\theta=25^{\circ}$ (cross), $\theta=38^{\circ}$ (circles). (A) Energy spectra after recons-
truction.

(B)

Real energy spectra observed because the two contributions from positive and negative angles

Fig. 5 : A schematic presentation of the evolution of kinetic energy as a function of the angle of observation of the fragment. 
According to the usual analysis made in terms of positive and negative angles $\left({ }^{18}\right)$, the high energy peak corresponds to the beginning of energy relaxation when $\Delta \theta$ is sma 11, and the low energy component is obtained after a longer relaxation time, corresponding to a larger $\Delta \theta$ because the rotation has evolved starting from the negative grazing angle, as shown schematically in figure 5 .

At larger angles, outside the grazing point, the high energy contribution has disappeared and also the width of the low energy component is greater. This is observed also for Sulfur isotopes, ${ }^{35} \mathrm{~S}$ showing both contributions although there is mainly a large hump around $45 \mathrm{MeV}$ for ${ }^{32} \mathrm{~S}$. However, even for ${ }^{34} S \mathrm{and}^{33} \mathrm{~S}$, where 6 and. 7 nucleons are missing, respectively, the partial damping appears very clearly. For much heavier systems, there is a much stronger angular focusing of deep inelastic phenomena. A very large relaxation is reached around a constant value after a rather small rotation angle, and sometimes the contribution from negative angles is negligeable. This has been discussed by Galin ${ }^{2}$ ) who introduced a

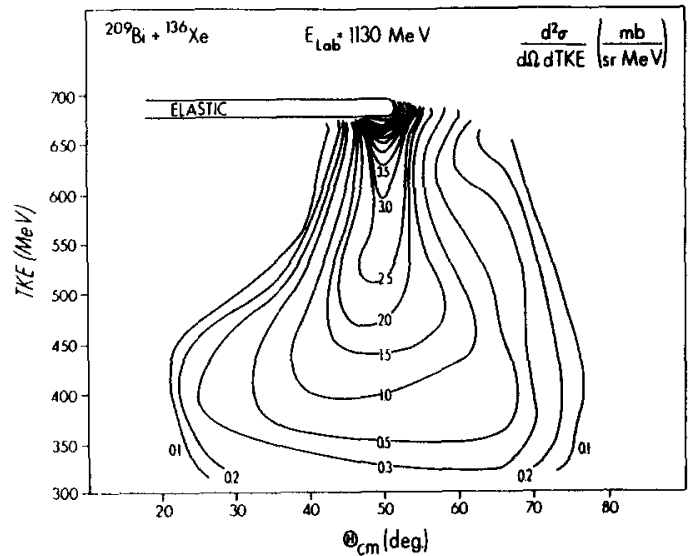

Fig. 6 : Energy damping in a very heavy system where the angular focusing is strong. Contour plots $\mathrm{d}^{2} \sigma / \mathrm{dEd} \theta$ for the deep inelastic products of the reaction $\left({ }^{136} \mathrm{Xe}+{ }^{209} \mathrm{Bi}\right)$ (ref. 21). modifjed Sommerfeld parameter, $n^{\prime}=\frac{Z_{1} Z_{2} e^{2}}{\hbar v^{\prime}}$, where the velocity $v^{\prime}$ is derived from $\left(E-B_{C}\right)$, the available energy above the barrier instead of the usual incoming energy. When $\eta^{\prime}$ is small (around 100-250) there is a wide rotation, while for large $\eta^{\prime}$ values, the strong damping occurs before a large rotation and the angular distribution is side peaking a little bit forwards the grazing angle. The focusing effect is so that, in a range of angles close to the grazing where most of the events occurs, one observes the entire energy spectrum from the elastic scattering value down to the lowest energy corresponding to the most relaxed events. Then the contour plot of $\frac{\mathrm{d} 2 \sigma}{\mathrm{dEd} \theta}$ appears like in figure 6 obtained $\frac{d \mathrm{dEd} \theta}{\text { by }}$ Schroeder et al $\left({ }^{21}\right)$ on $\left({ }^{209} \mathrm{Bi}+{ }^{136} \mathrm{Xe}\right)$.

\section{TOTAL RELAXATION - QUASI-EISSION.-}

The idea that the energy loss increases with the time of contact between

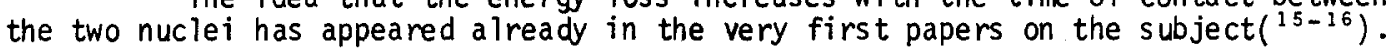
For large contact times, a limiting value of the total kinetic energy is expected to be reached. Experimental energy spectra as shown in figures 3 and 4 , exhibit a broad peak from which one may try to derive a mean value and a width at half maximum. For systems like $(A r+N i)\left({ }^{20}\right),(C a+N i)\left({ }^{22}\right),(C u+A u)\left({ }^{23}\right),(A r+A g)\left({ }^{24}\right)$, $(A r+A u)\left({ }^{25-26}\right)$, the mean kinetic energy decreases when the angle of observation decreases inside the grazing angle, and of course decreases even more at angles greater than the grazing angle since they correspond to long rotational times from trajectories issued from the negative side.

At the same time, the FWHM increases, as this could be expected if the relaxation phenomenon undergoes statistical fluctuations.

It is interesting to look at the limiting value for which we consider that there has been a complete relaxation. At least for all the cases where the 
bombarding energy does not exceed $10 \mathrm{~A} . \mathrm{MeV}$, except Xenon induced reactions at $9 \mathrm{~A} . \mathrm{MeV}$, the limiting value of the mean kinetic energy corresponds to a coulomb repulsion for a deformed liquid drop, i.e., it is definitively much lower than the Coulomb barrier for two touching spheres, or more precisely to the interaction barrier in the entrance channel.

The fact that the process leads into a scission configuration of two highly deformed fragments suggested that at least in the last stage, there is some similarity with nuclear fission. This is the reason why those deep inelastic events for which the relaxation is completed were called "Quasi-Fission" $\left({ }^{26}\right)$.

Like in fission, the kinetic energy is consistent with the Coulomb repulsion in the deformed Tiquid drop. It is independent on the entrance channel. There is a broad energy distribution around the mean value and also a rather broad mass distribution of the fragments. Unlike fission, the mass distribution of the fragments is very asymmetric and there is a strong remembrance of the projectile and target masses. The angular distribution does not follow the well known $1 / \mathrm{sin} \theta$ shape of ordinary fission, but exhibits generally a pronounced peak.

As an example of constancy of the quasi-fission energy, figure 7 pre-

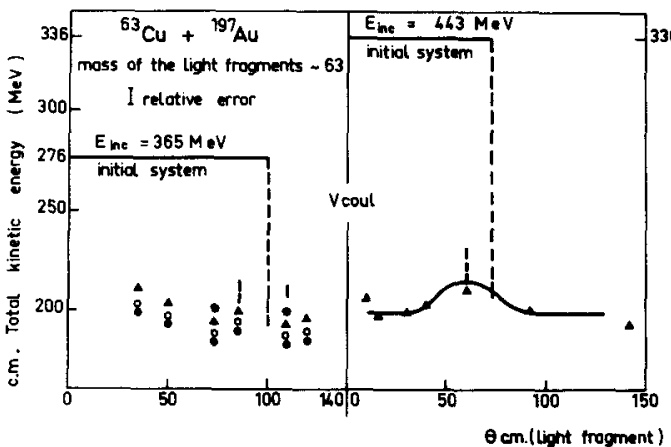

Fig. 7 : Mean value of the kinetic energy for quasi-fission events of masses around $\mathrm{A}=63$ in the bombardment of ${ }^{197} \mathrm{Au}$ by ${ }^{63} \mathrm{Cu}$ at two bombarding energies : 365 and 443 $\mathrm{MeV}$ (ref. 23). sents the results obtained in the system ${ }^{63} \mathrm{Cu}+\mathrm{Au}$ at two bombarding energies for masses around $A=63$. The mean value is equal to $205 \mathrm{MeV}$, while the interaction barrier is around $250 \mathrm{MeV}$. The observation that the mean value is independent of the bombarding energy is unexpected, since the ranges of angular orbital momenta involved in the two cases are quite different. Since the average value is around $50 \hbar$ at $365 \mathrm{MeV}$, and of the order of $120 \hbar$ at $443 \mathrm{MeV}$, the corresponding centrifugal energies should increase by a factor 5 . A possible explanation could be that the scission configuration and therefore the Coulomb repulsion depends on the angular momentum in such a way that the effect of centrifugal energy is compensated (shape more stretched for larger $\ell$ values).

The kinetic energies of the fragments are in principle very sensitive to the scission configuration and a precise comparison of coulomb energies to experiment should be made for each charge $Z_{3}$ of the lightest fragment. Since we know, from coincidence experimental results (9-1d) that the two-body break-up is associated with an excitation energy shared between the two fragments proportionnally to their masses, experimental kinetic energies can be corrected for particle evaporation. A comparison has been made $\left({ }^{22}\right)$ for fragments of different $Z$ values emitted in the reaction ${ }^{40} \mathrm{Ca}+{ }^{64} \mathrm{Ni}(182 \mathrm{MeV})$. The centrifugal term was calculated assuming sticking conditions, $i . e$. , the mean orbital angular momentum in the exit channel $\ell_{f} \hbar$ :

$$
\bar{l}_{f}=\bar{l}_{i} \frac{\mu R^{2}}{\mu R^{2}+\frac{2}{5} M_{1} R_{1}^{2}+\frac{2}{5} M_{2} R_{2}^{2}}
$$


In the moment of inertia of the composite system, $\mu R^{2}$, as we 11 as in the coulomb energy, $Z_{1} Z_{2} e^{2 / R}$, the distance between the two fragments is given by

$$
R=1.225\left(A_{1}^{1 / 3}+A_{2}^{1 / 3}\right)+d
$$

where $d$ is a free parameter simulating the deformation. Figure 8 a shows the agreement obtained with $d=4.5 \mathrm{fm}$ which is much larger than the usual liquid drop parameter. For a higher bombarding energy $\bar{l}_{j}$ goes up from 65 units at $80 \mathrm{MeV}$ to

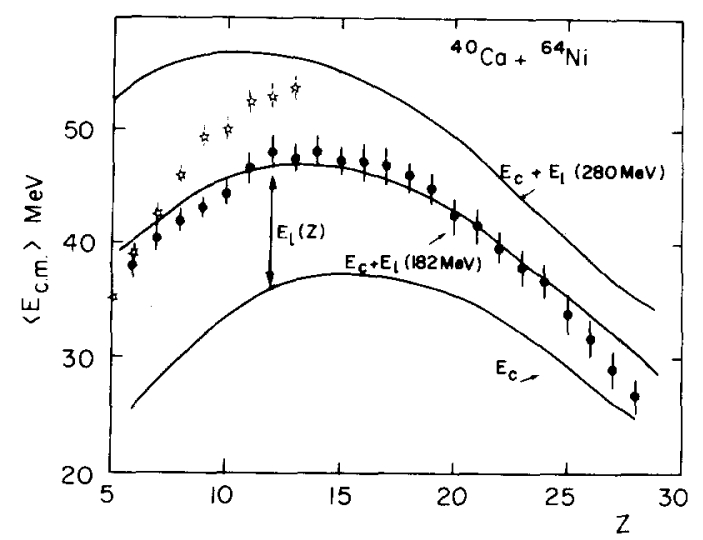

Fig. 8.a : Mean kinetic energies for the fully relaxed events (quasi-fission) of various $Z$. Dotted line : calculated energies assuming $E_{\text {cou } 1}+E_{\ell}$ for two bombarding energies : 182 and $280 \mathrm{MeV}$ for the system $\left({ }^{48} \mathrm{Ca}+{ }^{64} \mathrm{Ni}\right)$. Full points : experimental results at $182 \mathrm{MeV}$. Stars : experimental results at $280 \mathrm{MeV}$ (ref. 22).

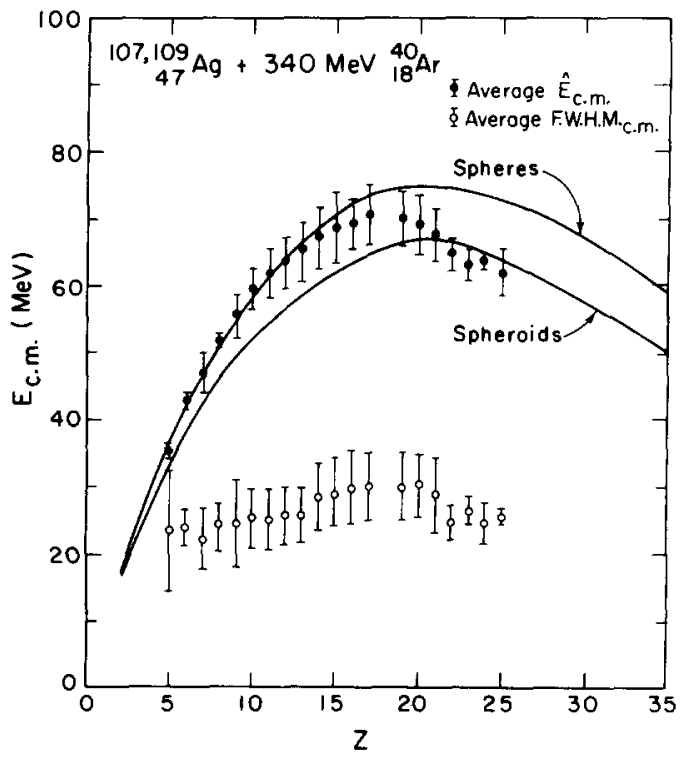

Fig. 8.b : Same data as in figure a for the system ( $\left.{ }^{40} \mathrm{Ar}+\mathrm{Ag}\right)$. Open circles indicate the FWHM (ref. 24).

$90 \mathrm{~h}$ units at $280 \mathrm{MeV}$. However, at least for light fragments $(Z<13)$ the experimental data do not show up the corresponding increase of centrifugal energy. A possible explanation could be that only the lowest $\ell$ values contribute to the very asymmetric splitting. The same type of analysis and comparison with Coulomb repulsion for two spheroids in contact has been made $\left({ }^{25-28-29}\right)$ on the systems $(\mathrm{Ar}+\mathrm{Ag})$, $(\mathrm{Ne}+\mathrm{Ag}),\left({ }^{40} \mathrm{Ca}+{ }^{40} \mathrm{Ca}\right)$. As far as the amount of kinetic energy which has to be dissipated was not too large $(50-300 \mathrm{MeV})$, it has been possible to observe a complete relaxation of the relative motion and to recognize a clear bell shape low energy component in the energy spectra from which one can extract an average kinetic energy. When the parameter $n^{\prime}$ is not very large, either because of small $Z_{1} Z_{2}$, or because of high velocity in the entrance channel, the complete relaxation is reached only after a rather large rotation. The spectra obtained at angles a bit forward the quarter-point angle look more complex than the example of figure 3 . The might appear two bumps, as this is quoted by Huizenga $\left({ }^{5-30}\right)$ for the system $\left({ }^{84} \mathrm{Kr}+165 \mathrm{Ho}\right)$ at $714 \mathrm{MeV}\left(\eta^{\prime}=201\right)$, for $\theta=19.7^{\circ}$ (figure 9). Here, the low energy bump corresponds to the negative angle contribution and the high energy bump corresponds to partially damped events since the rotation angle is very small. 

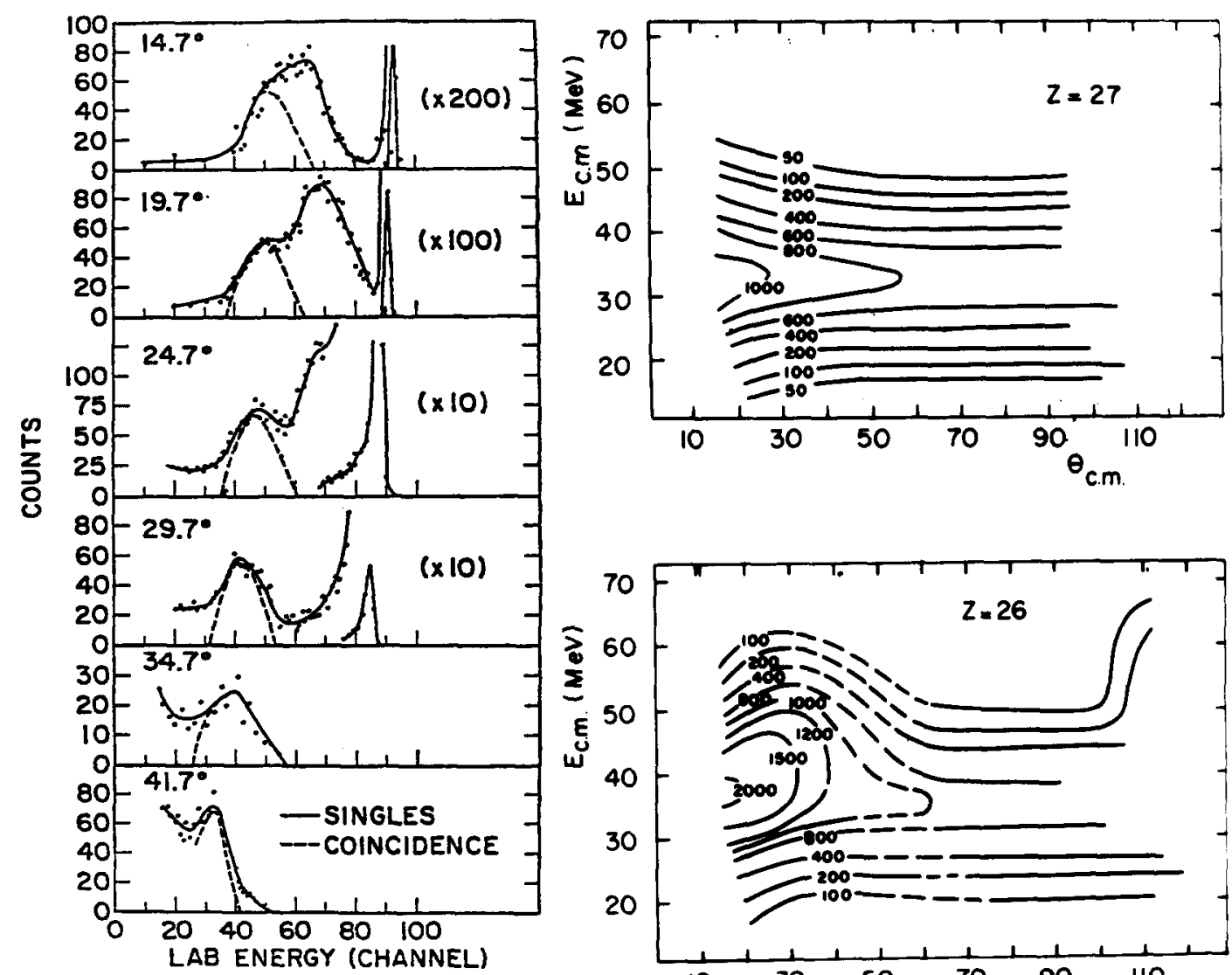

Fig. 9 : Energy spectra observed at various angles for krypton-like fragments in the system $\left({ }^{84} \mathrm{Kr}+165 \mathrm{Ho}\right)$ at $714 \mathrm{MeV}$. The 1 ow Fig. 10 : Contour lines, $\mathrm{d}^{2} \sigma / \mathrm{dEd} \theta$, for energy bump corresponds to negative angle fragments produced in the reaction and the higher energy bump corresponds to $\left({ }^{52} \mathrm{Cr}+{ }^{56} \mathrm{Fe}\right)$, in nearly symmetric splipartially damped fragments emitted at a tings (ref. 31). The completely relaxed positive angle very close to the grazing events present an isotropic angular disangle (figure reproduced from ref. 30). tribution.

This behaviour seems to me quite understandable and the quasi-fission events are still well indicated in the low energy bump which appears very nicely alone at larger angles where the only possible contribution is due to negative angles and therefore long rotation times during which complete relaxation can be reached. We want to show again three beautiful very recent illustrations of the validity of the fully relaxed kinetic energy justifying the name of quasi-fission which we developed $(4-23-27-26)$ a few years ago, and was criticized by Schroeder and Huizenga recentiy $\left({ }^{5}\right)$. The first is taken from the results $\left({ }^{31}\right)$ on a rather light and nearly symmetric system $\left({ }^{52} \mathrm{Cr}+{ }^{56} \mathrm{Fe}\right)$. Figure 10 shows Wilczynski's plot for a number of fragments around symmetric splitting. A projection of the contour plots on the energy ordinate would very nicely exhibit typical kinetic energies corresponding to fully relaxed events for each $Z$ value, as far as the region around and slightly inside the grazing angle is avoided. 
The same plot is shown in figure 11 for the system $\left({ }^{86} \mathrm{Kr}+{ }^{139} \mathrm{La}\right)$ at 2 bombarding energies ( $505 \mathrm{MeV}$ and $710 \mathrm{MeV}$ ). The results of Vandenbosch et al $\left({ }^{32}\right)$

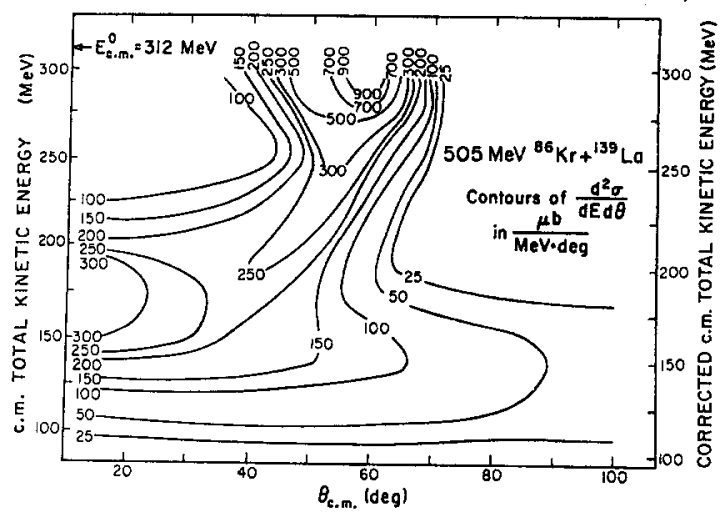
indicate complete relaxation at kinetic energies around a mean value close to $150 \mathrm{MeV}$ for the two cases. These events remain wi thout partially damped contribution when the centre of mass angle is taken far enough away from the grazing vicinity. The same thing was found $\left({ }^{3}{ }^{3}\right)$ on the system ${ }^{86} \mathrm{Kr}+{ }^{120} \mathrm{Sn}$ in G.S.I.

Another recent $\operatorname{data}\left({ }^{34}\right)$ is shown in figure 12 . There, projectile like fragments are counted in contour plots on both sides of the symmetry. Even at a velocity corresponding to $8.18 \mathrm{MeV} / \mathrm{n}, \mathrm{n}^{\prime}$ is large and the angular focusing is strong. There-

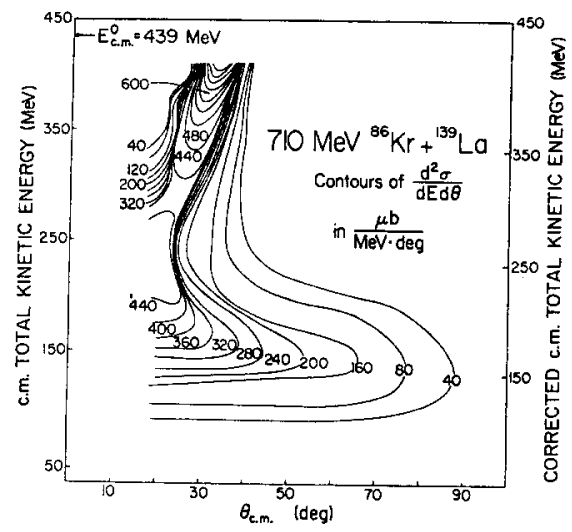
fore, there is a continuous decrease of $\frac{d \sigma}{d E}$ between very slightly inelastic events and the totally relaxed events. However, if one selects those cases closer to symmetry which correspond to important mass transfers, it appears clearly a distribution of kinetic energies around a mean value of $200 \mathrm{MeV}$. This corresponds to a Coulomb repulsion of two spheroids with axis ratios b/a between 0.6 and 0.8 , i.e., rather large deformations, even larger than at the scission shape in fission.

Fig. 11: Contour lines, $d^{2} \sigma / d E d \theta$, for fragments produced in the reaction $\left({ }^{86} \mathrm{Kr}+13{ }^{\mathrm{La}}\right)$ at 2 bombarding energies ( 505 and $710 \mathrm{MeV}$ ). Complete relaxation corresponds to $150 \mathrm{MeV}$ (ref. 32).

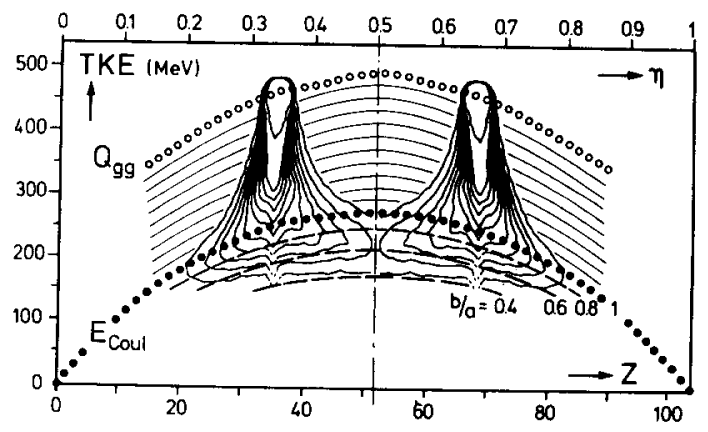

Fig. 12 : Contour lines, $\mathrm{d}^{2} \sigma / \mathrm{dEd}$, for fragments produced in the reaction $\left({ }^{86} \mathrm{Kr}+166_{\mathrm{Er}}\right)$ at $8.18 \mathrm{MeV} / \mathrm{amu}$. Qgg is calculated using the liquid-drop formula wi th shell corrections. The parallel lines between $E_{\text {coul }}$ and $Q_{g g}$ indicate the $20 \mathrm{MeV}$ TKE loss cuts. Ecoul has been calculated for two spheres using $\mathrm{r}_{\mathrm{o}}=1.2\left(\mathrm{~A}^{13}+\mathrm{A}_{2}^{13}\right)+$ $2 \mathrm{fm}$. Dashed curves indicate coutomb energies for elongated spheroids with $\mathrm{b} / \mathrm{a}=$ $0.8,0.6$, and 0.4 (from ref. 34). 
The three preceeding examples, added to dozen of published data, make us quite confident in the idea that the composite systems when they have been formed can live until a complete relaxation of the kinetic energy and there is some kind of pile-up at the kinetic energy corresponding to coulomb repulsion at the scission of two fragments at rest, like in the fission process. This concept is quite important since it may help us to understand how the shape of the composite system behaves between the contact point and the final disruption.

Nevertheless, with very large bombarding energies combined wi th very heavy projectiles, $7 i k e{ }^{136} \mathrm{Xe}$ beams at $1130 \mathrm{MeV}(8.5 \mathrm{MeV} . \mathrm{A})$, on ${ }^{209} \mathrm{Bi}$, the very wide bump of the low kinetic energy contribution has a maximum( ${ }^{21}$ ) at some $70 \mathrm{MeV}$ above the entrance channel Coulomb energy (see figure 13.a). This means that complete relaxation is not reached. At the same velocity, with $718 \mathrm{MeV}$, Krypton ions

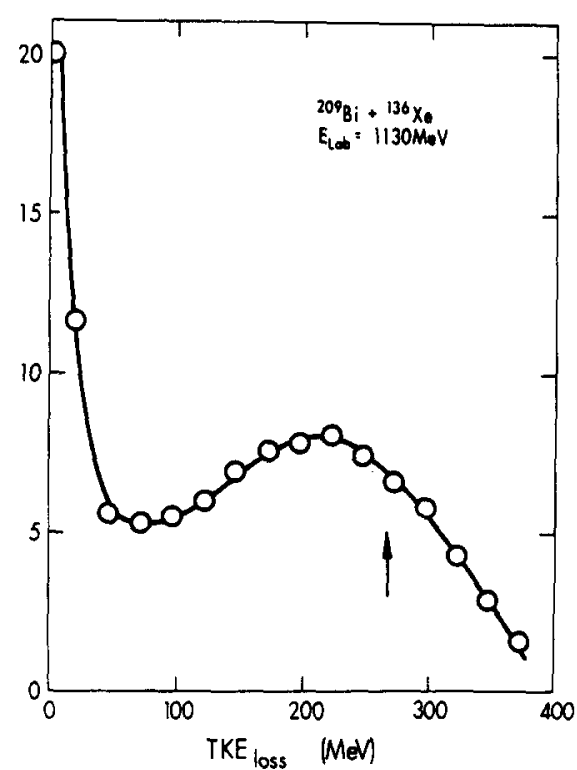

Fig. 13.a : A case where complete relaxation is not achieved : $\left({ }^{136} \mathrm{Xe}+{ }^{289} \mathrm{Bi}\right)$ at 1130 $\mathrm{MeV}$. The maximum of the bump of kinetic energy is located at $70 \mathrm{MeV}$ above the entrance channe1 coulomb energy (ref. 21).

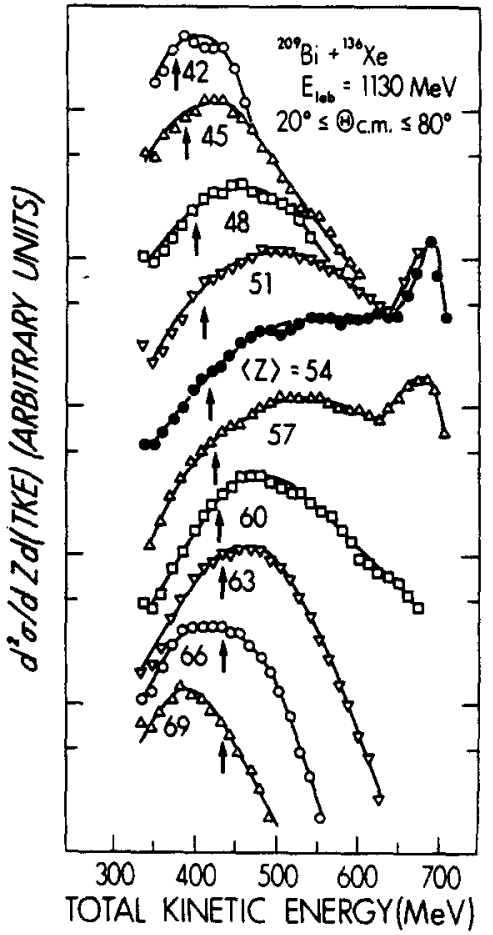

Fig. 13.b : TKE spectra for various $Z$ values.

bombarding ${ }^{209} \mathrm{Bi}$, Huizenga et al $\left({ }^{35}\right)$ have found lower kinetic energies corresponding to Coulomb energies of deformed shapes. One of the possible explanation for the difference is that the total amount of energy to be dissipated in order to attain the Coulomb energy is greater in the case of (Xe $+B i$ ) than in the case of $(\mathrm{Kr}+\mathrm{Bi})$. This seems indeed to be verified by the results $\left({ }^{6}\right)$ obtained at a smaller bombarding energy ( $900 \mathrm{MeV}$ ) where the maximum of the totally relaxed bump was found at a lower energy than the Coulomb repulsion. But here the projectile kinetic energy loss necessary to reach the Coulomb repulsion was less than $100 \mathrm{MeV}$, while it was $300 \mathrm{MeV}$ for an incident energy of $1130 \mathrm{MeV}$. Also $\mathrm{Z}_{1} \mathrm{Z}_{2}$ is higher and the Coulomb forces may keep the colliding system more apart so that there will be less deformation and fission-like configurations would be obtained only in a few cases. As a matter fact if only fragments quite heavier than Xenon are considered, 
for example with $\langle Z\rangle$ greater than 66 , i.e. for large $Z$ and $A$ transfers, the kinetic energy loss bump peaks at a much smaller value, around $350 \mathrm{MeV}$, which corresponds to low Coulomb repulsions of deformed shapes.

\section{THE WIDTH OF THE KINETIC ENERGY DISTRIBUTION OF QUASI-FISSTON EVENTS.}

In all the cases where the kinetic energy of the fragments was compared to calculated Coulomb repulsions, this was done for the value measured at the maximum of the low energy bump. The width of this bump is rather large and it is not a lways very easy to extract the FWHM, particularly because, on the high energy side there is the contribution of partially damped events. However it is interesting to notice $\left({ }^{24}\right)$ that for figures 8 and 14 as well as for all the cases where it has

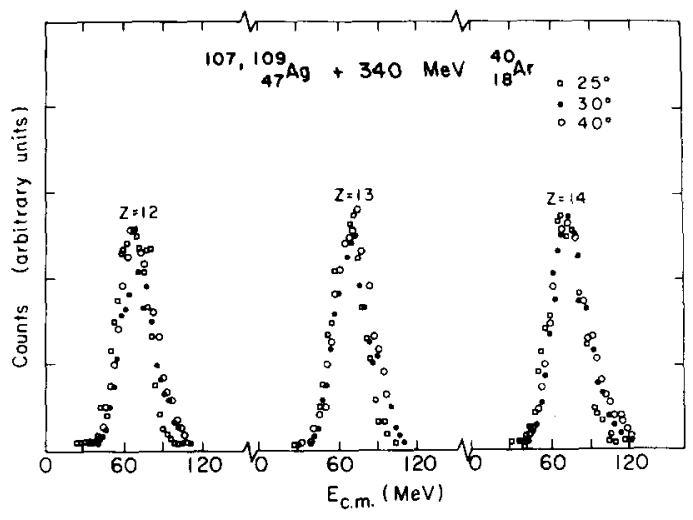

Fig. 14 : Width of the quasi-fission fragment kinetic energies. The kinetic energy distribution has been measured for several fragments at different angles in the system $\left({ }^{40} \mathrm{Ar}+107,109 \mathrm{Ag}\right)($ ref. 24$)$. been considered, the width does depend neither on the bombarding energy nor on the angle of observation or on the $Z$ of the fragment as far as the region around the grazing angle is avoided. This demonstrated the statistical aspect of the energy loss which is submitted to important fluctuations like in the fission process. One might think that they represent not only fluctuations in the shape at the scission point, but also variations in the centrifugal contribution since many $\ell$-waves are involved. It is quite remarquable that the kinetic energy, particularly for light fragments, is going down to very low values (fig. 15-16). They correspond, in terms of coulomb repulsion, to very deformed shapes, which have no real physical significance. Rather, they should be considered as resulting from fluctuations in the process of energy dissipation between the relative motion macroscopic degree of freedom and the intrinsic degrees. When the fragment has a small number of nucleons, the relative contribution of the momentum distribution of individual particles is much bigger and one might explain qualitatively why the kinetic energy of nitrogen and carbon fragments is so small as shown $\left({ }^{31}\right)$ on the Wilczynski's plots of figure 16. Before to finish on this question of fully damped phenomena, I should like to raise again the problem of the estimates of the angular momentum contribution to the exit channel energy. It is indeed rather uneasy to deduce what is the magnitude of the centrifugal component, i.e., $\frac{\ell_{f}\left(\ell_{f}+1\right) \pi^{2}}{2 J}$, because

i) the range of incident $\ell$-waves which contribute to the totally relaxed events is not so well known,

ii) the sticking limit asumption is not totally proved,

iii) the moment of inertia depends on the deformation, which may itself be deduced from the Coulomb energy. 

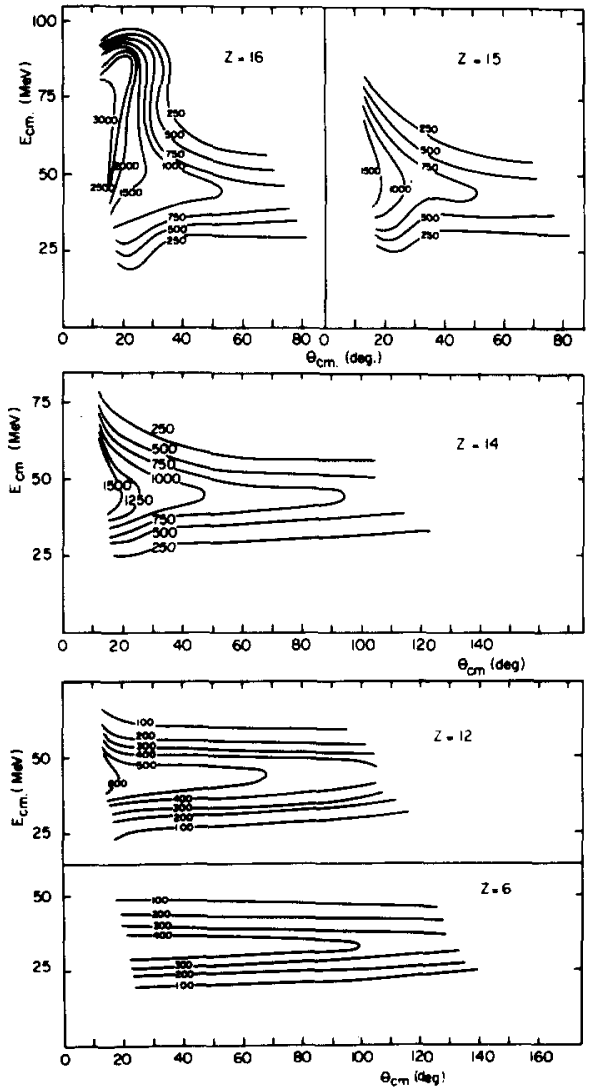

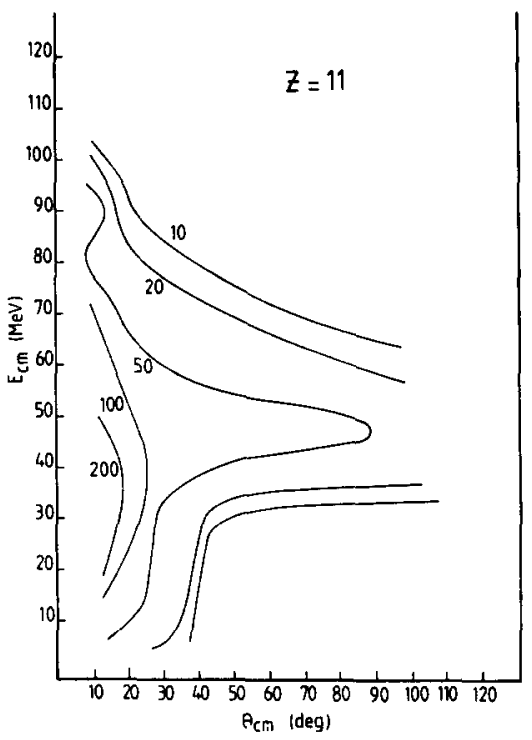

Fig. 16 : Contour plot $d^{2} \sigma / d \bar{E} d \bar{\theta}$ for the fragment $Z=11$ in the reaction induced by 160 on ${ }^{92}$ Mo at a c.m. bombarding energy of $157 \mathrm{MeV}$. At forward angles the kinetic energy distribution of the fragment exhibits a very broad spectrum down to $10 \mathrm{MeV}$ (ref. 31).

Fig. 15 : An example of the observation of complete relaxation at backward angles for fragments around $Z=25$ produced in the reaction $\left({ }^{40} \mathrm{Ar}+{ }^{58} \mathrm{Ni}\right)$ (ref. 53$)$.

However, when the centrifugal energy is only a small contribution as compared to the coulomb repulsion, the possible error made on its estimates is not so relevant. But for light systems and high angular momenta, it could be of the same magnitude, like in Cormier et al's experiments $\left({ }^{36}\right)$.

In connection with this centrifugal energy contribution, a very interesting result has been obtained $\left({ }^{37}\right)$ recently, on a light system $\left({ }^{32} S+{ }^{27} \mathrm{Al}\right)$ at $175 \mathrm{MeV}$. In the energy spectra of fragments, observed at angles where complete relaxation is achieved, a low energy bump appears on the gaussian shape, which might indicate that two different dynamic equilibria are attained in the scissioning system. Furthermore, coincidence measurements between the two products are quite convincing that two groups of events corresponding to two different kinematics are appearing. Figure 17 illustrates the two peaks for the coincidence probability when the detectors were places at $-30^{\circ}$ and $+34^{\circ}$. Events were selected so that $\left(A_{1}+A_{2}\right)>56$ in order to minimize the effect or particle emission on the kinematics. This kind of two peak curve ressembles the usual energy spectra obtained in fission but here the low energy peak is higher. Assuming a two body break-up from the composite, $A=59$, the kinematics requires a $Q$ value near - $27 \mathrm{MeV}$ for fragment masses near 20 (high energy peak around $85 \mathrm{MeV}$ ) while the low energy peak at $55 \mathrm{MeV}$ corresponds to a $Q$ value of around $47 \mathrm{MeV}$, for masses near $A_{1}=35$ (see part $b$ of 

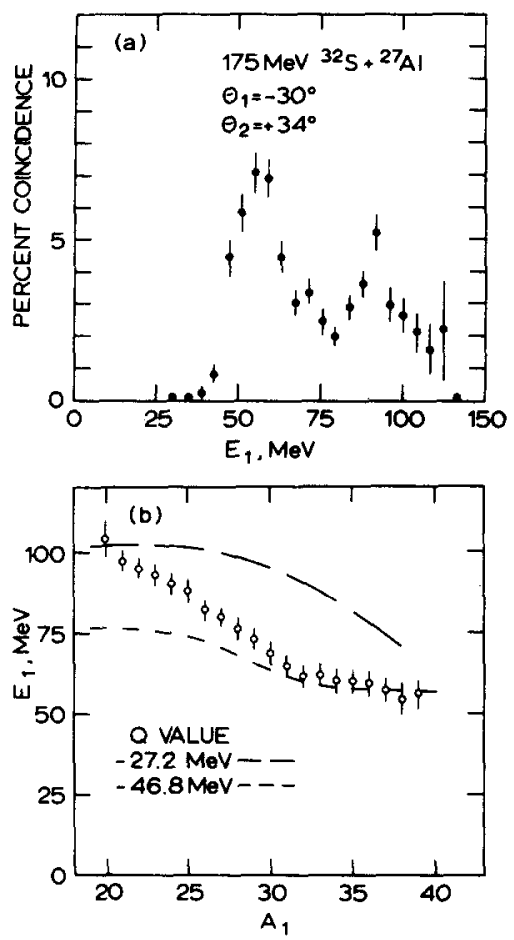

Fig. 17 : Fragment coincidences observed at $30^{\circ}$ and $34^{\circ}$ on opposite sides of the beam, in the system ${ }^{32} \mathrm{~S}+{ }^{27} \mathrm{Al}$. a) percent coincidences versus kinetic energy of the product detected at $30^{\circ}$. b) mass variation of the most probable kinetic energy of the products. The lines show the variation predicted for two-body break up with the indicated $Q$ values $\left(A_{1}+A_{2}\right) \geqslant 56$ (from ref. 37).

figure 17). There is therefore a clear demonstration of the existence of two separate two-body processes which differ in most probable $Q$ value by some $20 \mathrm{MeV}$. The explanation suggested by the authors is that the two groups of scissioning nuclei have different average angular momenta. Assuming a saddle point shape of ${ }^{5} \mathrm{Cu}$ calculated with the liquid drop model, the authors have calculated the expected variation of the total kinetic energy as a function of the angular momentum. They conclude that for the higher $Q$ value, only low $\ell$-waves contribute (lower than $15 \hbar$ ) whereas for the higher kinetic energy an average angular momentum of $48 \mathrm{n}$ contributes to the centrifugal energy. This might be evidence for the existence of a low $l$ branch for deep inelastic reactions, and a $l$ window for complete fusion.

\section{INCOMPLETE RELAXATION. TRENDS BETWEEN QUASI-ELASTIC SCATTERING AND COMPLETE ENERGY DISSIPATTON. CORRE LATION WITH ANGULAR MOMENTUM DISSIPATTON.-}

It is clear that the dissipative processes lead to continuous kinetic energy distributions connecting the quasi-elastic events to the domain of full relaxation (quasi-fission). And there is a great interest to study with more details the behaviour of the energy loss when time evolves.

How fast is the energy dissipation, how is it related to nucleon exchange, angular momentum transfer, charge distribution? How far can experimental data help the choice of a theoretical model of energy dissipation? Since the first contour diagrams published by Wilczynski( $\left.{ }^{18}\right)$, the picture of two ridges in the $\theta$ versus $E$ space is well known. The first one starts at the quasi-elastic scattering energy and is moving to forward angles while the kinetic energy is decreasing. The second seems to start at zero degree and moves to larger angles with a decrease in energy till it reaches the totally relaxed value. As a matter fact it represents the continuation to negative angles of the first peak, as explained already in figure 5 . 
However, for heavy systems, and for large $\eta^{\prime}$ values, the deflection function $\theta=f(l)$ does not show any plunging branch so that the contribution to small angles inside the grazing does not appear. There is a strong angular focusing effect and one cannot follow the energy loss along the $\Delta \theta$ coordinate and use relation (2). We have already seen pictures of this type (figure 6 ).

Kinetic energy is $\operatorname{known}\left({ }^{38}\right)$ to be lost at least for a large part through nucleon exchange between the two partners. Therefore, an interesting way to examine the energy dissipation mechanism consists in studying the correlation between energy loss and nucleon exchange.

On a theoretical basis( $\left.{ }^{41}\right)$, the one-body energy dissipation has been calculated by Randrup $\left(^{42}\right)$ with the proximity formalism, for target and projectile nuclei with half density radii $R_{T}$ and $R_{p}$, and diffuseness $d$ :

$$
\left.-\frac{d E}{d t}=4 \pi \frac{N_{0}}{\mu} \frac{R_{T} / R_{p}}{R_{T}+R_{p}} \quad \text { d.EP( } \xi_{0}\right)
$$

where $N_{0}$ is the transfer flux density around $2.510^{-23} \mathrm{MeVs} \mathrm{fm}^{-4}$ and $\varphi\left(\xi_{0}\right)$ is a universal flux function at a separation distance $\left(\zeta_{0}\right)$.

For $\xi_{0}$ between $-0.75 \mathrm{fm}$ and $+0.75 \mathrm{fm}$, the flux function $\left(\xi_{0}\right)$ varies around 1.2, and $\mathrm{dE} / \mathrm{dt}$ is estimated as $\mathrm{dE} / \mathrm{dt} \approx 10^{21} \mathrm{sec}^{-1} . \mathrm{E}$, for medium mass projectiles and heavy targets. Roughly the time for $100 \mathrm{MeV}$ of energy relaxation is of the order of $10^{-21} \mathrm{sec}$. However, this is an estimate with no particular account of angular momentum.

The typical pieces of informations to be explored are, according to Huizenga et al $\left({ }^{35}\right)$, the charge distributions for various bins of energy loss, and the plot of the kinetic energy loss versus the distribution width. A classical example is shown in figures 18 and 19 for the system $\left({ }^{209} \mathrm{Bi}+{ }^{136} \mathrm{Xe}\right)$. The width
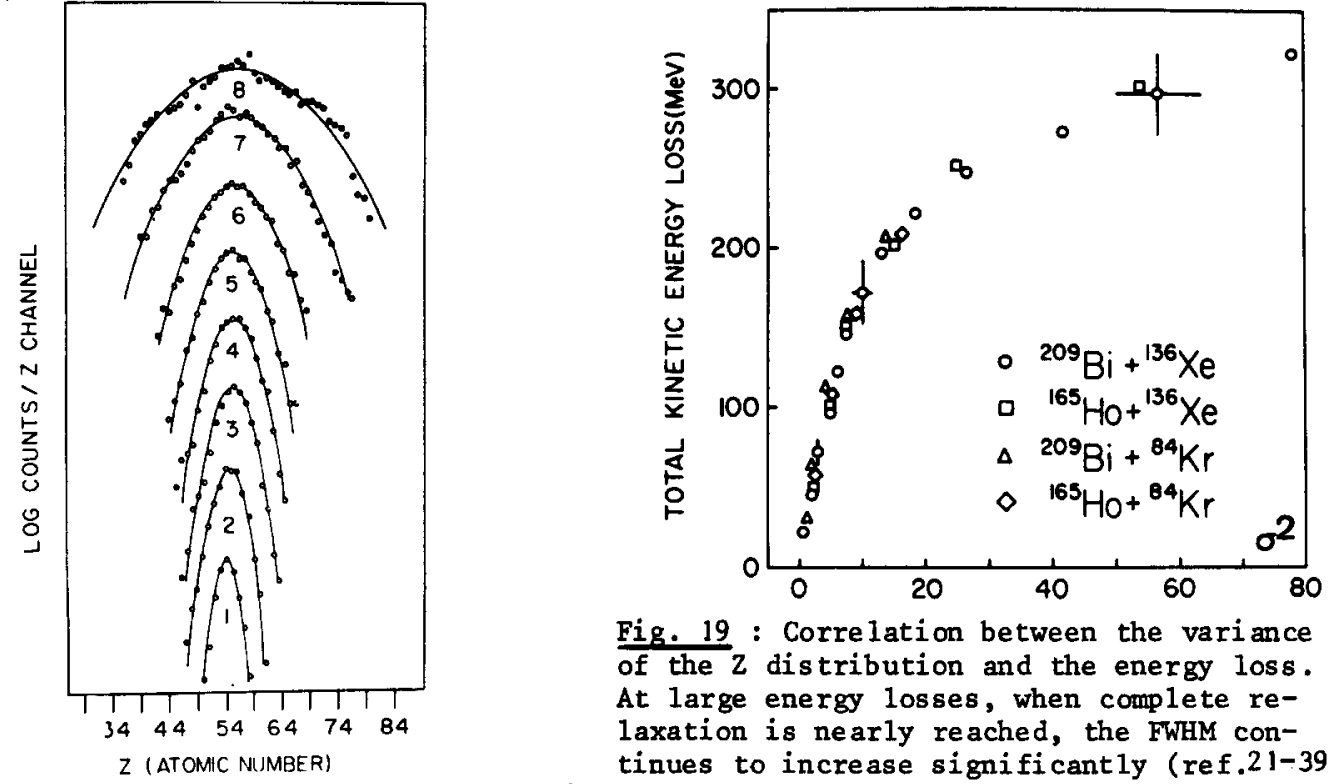

Fig. 19 : Correlation between the variance of the $\mathrm{Z}$ distribution and the energy loss. At large energy losses, when complete relaxation is nearly reached, the FWHM continues to increase significantly (ref.21-39)

Fig. 18 : Charge distributions for various

bins of TKE loss for the projectiles-like

fragments in the reaction $\left({ }^{136} \mathrm{Xe}+209_{\mathrm{Bi}}\right)$

(ref.39). 
increases with the energy loss, suggesting indeed very strongly a diffusion process. The correlation between energy loss and variance of the $Z$ distribution has been derived by Schroëder et $a 1^{2}\left(^{1-39}\right)$. Starting with the available kinetic energy in excess of the Coulomb energy, $E=\left(E-B_{C}-\right.$ TKE loss), they write the relation between energy loss and time evolution,

$$
\frac{d E}{d t} e x=-\frac{m}{\mu} E \frac{d N}{d t}
$$

if the nucleon flux between the two colliding nuclei, $\frac{d N}{d t}$, is the only process for dissipation.

The total energy dissipated during the time interval necessary to exchange one nucleon is

$$
\delta E_{e x}=-\frac{d E}{d N} \text { ex }=\frac{m}{\mu} E
$$

and the correlation between energy loss and variance of the $Z$ distribution

$$
\begin{aligned}
& T_{\text {TKE }} \text { loss } \\
& =E-B_{C}-E=\left(\bar{E}-B_{C}\right)\left(1-\exp \left(-\frac{m}{\mu} N\right)\right) \\
& T_{K E_{\text {los }}}=\left(\bar{E}-B_{C}\right)\left(1-\exp \left(-\frac{m}{\mu} \frac{A}{Z} \sigma_{Z}{ }^{2}\right)\right.
\end{aligned}
$$

assuming $N=\frac{A}{T} \sigma_{Z}{ }^{2}$, since it is known that the equilibration of mass to charge asymmetry degree of freedom occurs very shortiy.

Experimental results for the energy loss per nucleon exchanged, $\delta E$, have been plotted $(39-40)$ according to expression (7), for several systems at a bombarding energy of $8.5 \mathrm{MeV} . A$, The values of $\delta E$ were obtained by differentiating curves of energy loss versus $\sigma_{7}{ }^{2}$ (related to $N$ ) extracted of the results reported like in figure 20 . The linear $Z$ relationship was well fitted, with the same slope for the three systems ${ }^{84} \mathrm{Kr}+{ }^{165} \mathrm{Ho},{ }^{136} \mathrm{Xe}+{ }^{165} \mathrm{Ho}$ and ${ }^{136} \mathrm{Xe}+{ }^{206} \mathrm{Bi}$. However for ${ }^{84} \mathrm{Kr}+{ }^{197} \mathrm{Au}$, the results indicated by Moretto $\left({ }^{3}\right)$ at $7.2 \mathrm{MeV} . \mathrm{A}$, correspond to a different slope.

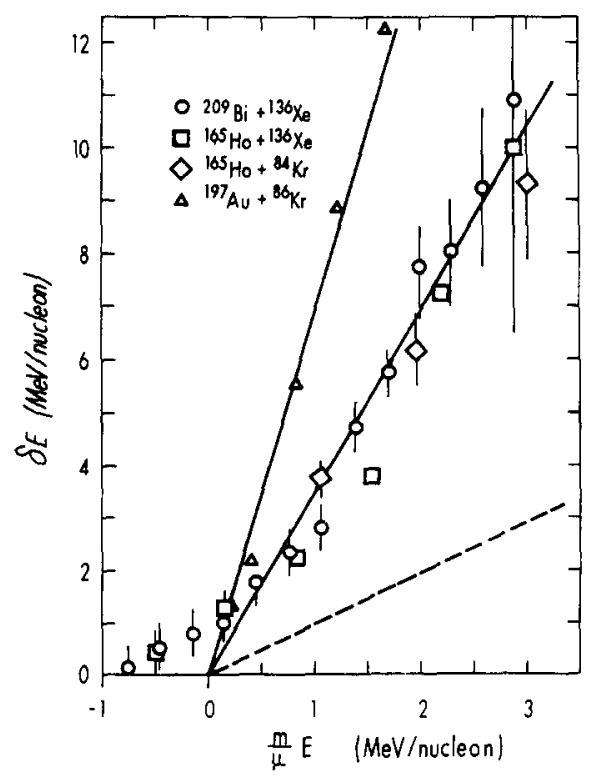

Fig. 20 : Total kinetic energy $\delta$ E dissipated per nucleon exchanged as a function of the available energy per nucleon $\frac{m}{H}$. The solide lines are drawn through the data points. The dashed line represents the amount of energy dissipated by the exchange process itself (eq. 7).(ref. 39). 
The deviation for small energies, $i . e$. at nearly complete relaxation, indicates highly deformed intermediate systems so that the Coulomb energy is lower than the one put in the calculation of TKE loss and of the abscissa $E=0$.

Now, considering the straight lines of figure 20 , the comparison with equation (7), indicates that the mass exchange field process can account for only $30 \%$ of the energy dissipation. Since the experimental slopes are greater, an additional mechanism following the same dissipative law, should be responsible for the energy loss. This might be for example particle-hole excitation which is still a one-body process. Nucleon exchange is certainly responsible for a fraction of the total energy loss, but not a11. This fraction is not yet very we 11 known and depends probably on the bombarding energy. Rudolf et al $\left({ }^{43}\right)$ have found for $\left({ }^{86} \mathrm{Kr}+{ }^{166} \mathrm{Er}\right)$ at $8.2 \mathrm{MeV}$.A, a rather good agreement between experimental $\sigma_{7}{ }^{2}$ for various energy losses and a one-body calculation. At smaller bombarding energies $(6.2 \mathrm{MeV} . \mathrm{A})$, the agreement is not so good. But Vandenbosch et al $\left({ }^{32}\right)$ did find for the system ( ${ }^{86} \mathrm{Kr}+{ }^{\left.1{ }^{39} \mathrm{La}\right)}$ that the energy loss due to the particle transfers accounts for less than half the observed energy loss.

\section{ANGULAR MOMENTUM TRANSFER AND ENERGY DISSIPATION}

The simple treatment sketched above do not take account explicitely of the angular momentum transfer. It describes indeed the energy loss as a whole, but the transfer of orbital angular momentum into intrinsic spin should obviously decrease the centrifugal energy in the exit channe1. Therefore both radial and tangential energy dissipation should be included.

A very nice picture of the monotonic correlation between energy loss and angular. momentum transfer has been furnished already in 1974 by Gross et al $\left({ }^{4} 4\right)$, using classical dynamics and the friction force concept. In many case, the deflection function allows a simple relation between the angle and the interaction time and therefore one may use expression like equation (2). But this is not always the case, particularly for heavy systems where a double rainbow appears $\left({ }^{45}\right)$ or when a very strong angular focusing effect inhibits the observation of a time evolution as a function of the angle of emission. However, one can still use the kinetic energy loss as a clock, and make the asymption that there is a monotonic increase in the total kinetic energy loss with decreasing values of the impact parameter $\left({ }^{46}\right)$.

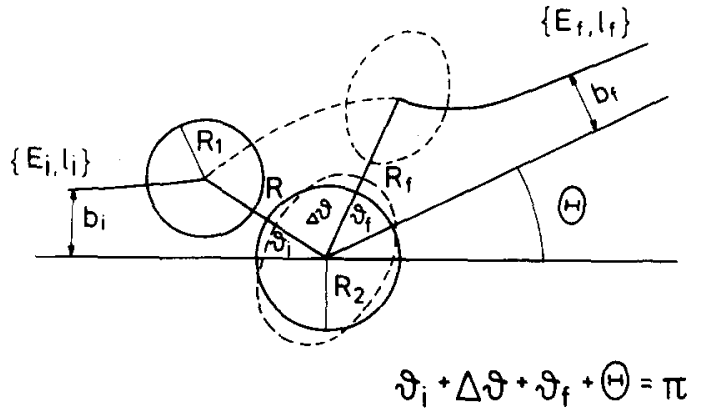

Fig. 21 : Schematic illustration of the calculation of the coulomb deflection functions and of the influence of nuclear interaction. From Nörenberg et al. (ref. 47).
For a given value of $\ell_{i}$, the incident angular momentum, the interaction time $\tau\left(\ell_{i}\right)$ is determined by

$$
\hbar \ell_{j} \cdot \tau\left(\ell_{i}\right)=\Delta \theta\left(\ell_{j}\right) \mathbf{J}(\ell)
$$

where $Y$ is relative moment of inertia of the double-nucleus system having an orbital angular momentum related to $\ell_{i}$ according to the clutching conditions (sticking or no sticking). Then figure 21 due to Nörenberg $\left({ }^{4}\right)$ shows that :

$$
\Delta \theta\left(l_{j}\right)=\theta_{c}\left(l_{j}\right)-\theta_{\exp }\left(l_{i}\right)
$$

$\theta_{\exp }\left(\ell_{j}\right)$ is the actual angle where the fragment is detected, $\theta_{c}\left(l_{j}\right)$ is the coulomb deflection angle

$$
\theta_{c}\left(\ell_{i}\right)=180^{\circ}-\theta_{c i}-\theta_{C f}
$$


$\theta_{c i}$ and $\theta_{c f}$ are the coulomb angle in the incident and final channels, respectively

$$
\theta_{c i f}=\arccos \left(1+\frac{2 E l_{i f}{ }^{2}{ }^{2}}{\mu\left(Z_{p} Z_{T} e^{2}\right)^{2}}\right)^{1 / 2}-\arccos \left[\frac{\left(R_{p}+R_{T}\right)+\frac{l_{i f}{ }^{2} \hbar^{2}}{\mu Z_{p} Z_{r} e^{2}}}{\left(R_{p}+R_{T}\right) 1+\left(\frac{2 E \ell_{i f}{ }^{2} \hbar^{2}}{\left(Z_{p} Z_{T} e^{2}\right)^{2}}\right)^{1 / 2}}\right]
$$

Coming back to relation (11), the problem is now to find a connection between bins of $\ell$ values and bins of kinetic energy losses. This was done in a very simple manner assuming a monotonic increase in the total kinetic energy loss with decreasing impact parameters $\left({ }^{46}\right)$. Then applying the sharp cut off approximation, $i . e$. ,

$$
\sigma_{\ell}=\pi t^{2}(\ell+1)^{2}
$$
the cross section for events between $E_{k}$ and $E$, and consequently between $\ell_{k}$ and $\ell_{\ell}$
is

$$
\Delta \sigma_{i j}=\pi \dot{\pi}^{2}\left(\left(\ell_{\ell}+1\right)^{2}-\left(\ell_{k}+1\right)^{2}\right.
$$

So, one can draw a deflection function from the kinetic energy loss spectrum and the difference $\Delta \theta(l)$ calculated according to equation (11). An example is shown in figure 22. The difference between the Coulomb deflection function and the actual deflection function derived from the TKE loss, $\Delta \theta(\ell)$, appears clearly in figure 23.

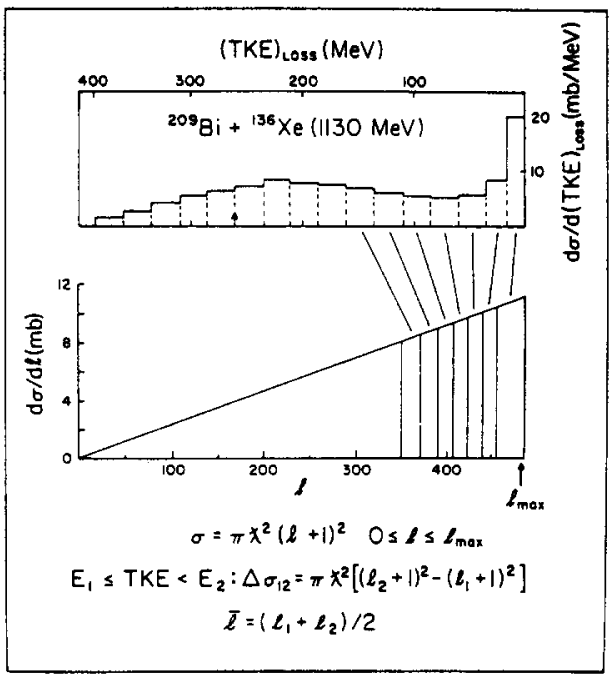

Fig. 22 : Derivation of a deflection function from the kinetic energy loss (system $\left.136 \mathrm{Xe}+{ }^{209} \mathrm{Bi}\right)($ ref. 60$)$.

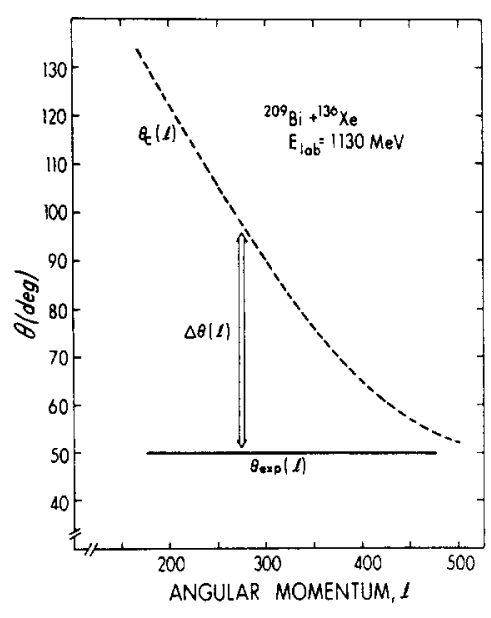

Fig. 23 : Difference between the coulomb deflection function and the deep inelastic deflection function (system ${ }^{136} \mathrm{Xe}+{ }^{209} \mathrm{Bi}$ ) (ref. 60). 
The angular momentum dependent interaction times are deduced from equation (10). They were found to follow the empirical relation

$$
\tau(\ell)=\tau_{0} \exp (-\beta \ell)
$$
with a value of $B$ depending on $\ell_{\text {max }}, A_{T}$ and $A_{P}, Z_{T}$ and $Z_{p}$. For the system
$(X e+B i)$, Schroëder et al $\left({ }^{46}\right)$ found that $\tau_{0} P_{S}$ of the ofder of $2.10^{-20} \mathrm{sec}$ assuming a sticking model. This is rather a long time as compared to the theoretical calculation using equation (5). But one should notice that the totally flat deflection function of figure 23 is probably not correct. One would rather expect a double rainbow and a rising branch for low $l$-waves. Furthermore both fluctuations in energy loss and in angular momentum transfer interfer so that many reaction paths lead to the same angle and to the same bin of energy loss. There is no particular reason to divide the energy spectrum in vertical cuts corresponding to vertical bins.

A more sophisticated treatment has been done by Wolschin and Nörenberg $\left({ }^{48}\right)$. The very important point is that they consider three distinct approaches contributing to the kinetic energy damping process : a radial dissipation following a one-body procedure, a tangential dissipation corresponding to angular momentum transfer during the clutching of the two nuclei, and a deformation which develops before scission at a later stage. The transport theory and the fluctuations deriving from the statistical assumptions are applied to the three degrees of freedom - radial motion - rotational motion - deformation. Moreover the deflection function is determined from the angular distribution as followed:

The deflection angle for an impact parameter $b$ is obtained by two contributions, $\Theta_{c}(b)$ the coulomb deflection function, and $\Theta_{N}(b)$, the nuclear part. (b) is calculated as explained in figure 21 , and the nuclear part is put in a parametrized form

$$
\Theta_{N}(b)=B \Theta_{C}^{g r} \frac{b}{b_{g r}}\left(\frac{\delta}{\beta}\right)^{b / b} g r
$$

where $\delta$ is for the deviation of the Coulomb trajectory near the grazing angle $\theta_{C}^{g r}$, and $B$ is for the main deviation when $b<b_{g r}$.

By iteraction, $\beta$ and $\delta$ are adjusted to fit the experimental angular distribution, assuming classically

$$
\frac{d \sigma}{d \theta}=2 \pi \sum_{n} b_{n}\left|\frac{d b}{d \theta}\right|_{b}=b_{n}
$$

Large $\beta$ correspond to small $\eta^{\prime}$ parameters and rotations towards negative angles. Small $\beta$ are for large $\eta^{\prime}$, angular focusing and therefore a rather small deviation from the Coulomb deflection.

Figure 24 shows that the deflection function presents a rising branch at low impact parameters, even for $\mathrm{Xe}+\mathrm{Bi}$ at $8.3 \mathrm{MeV} / \mathrm{amu}$. A comparison with the picture given in figure 23 indicates that $\Delta \theta$ values can differ strongly and low $\ell$-waves trajectories do not go into angles inside the grazing. Furthermore, large kinetic energy losses might not be associated so strictly to low l-waves. As a matter fact gamma multiplicity measurements have shown $\left({ }^{50}\right)$ that for the most relaxed events, Mr is as large as for partially damped events and there is no evident effect of an eventual decrease of intrinsic angular momentum when the kinetic energy loss is the greatest. This appears very clearly in figure 25 where gamma multiplicities, which are directly connected to the angular momenta shared by the fragments, were measured at different TKE. If there was a univoque correspondance between low kinetic energies and low impact parameters and therefore low intrinsic spins in the fragments, as expressed in the histogram of figure 22, Mr should 


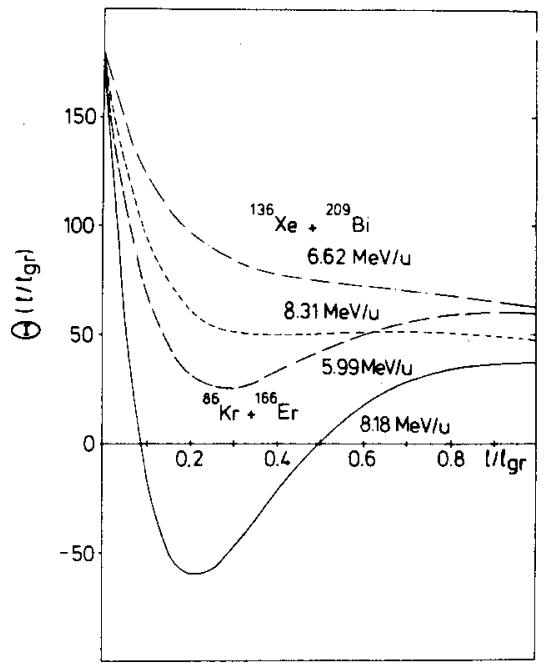

Fig. 24 : Theoretical calculations 61 for mean deflection functions of several systems, obtained by coupling to the $\Delta \theta$ equation (39) the kinetic energy equation (41). Sys tems $\left({ }^{86} \mathrm{Kr}+{ }^{166} \mathrm{Er}\right)$ and $\left({ }^{136} \mathrm{Xe}+{ }^{209} \mathrm{Bi}\right)$ at two bombarding energies.

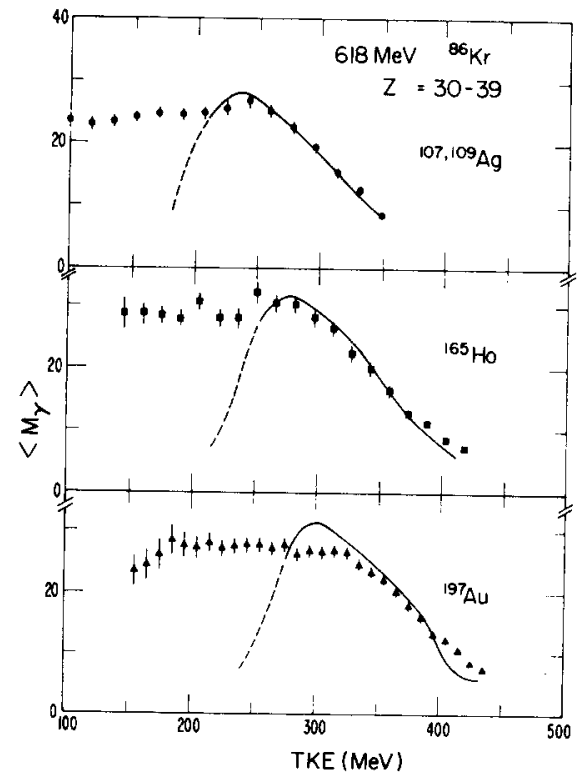

Fig. 25 : Gamma multiplicity measurements for various amounts of total kinetic energy loss. $\mathrm{M}_{\gamma}$ is more or less constant over a wide range of TKE (ref. 63). 
decrease strongly for the smallest TKE. On the contrary, calculations made according to ref. 62 indicate that there are still more than $100 \hbar$ lwaves contributing to energy losses around $250 \mathrm{MeV}$.

Now we can try to find a relation between $\Delta \theta$ expressed in (11) and the interaction time.

There will appear three relaxation times, $\tau_{R}$ for the radial kinetic energy dissipation, $\tau_{\alpha}$ for the deformation relaxation and $\tau_{l_{j}}$ for the interaction determined by the rotation of the composite system through the angle $\Delta \theta\left(l_{i}\right)$.

From the study of transport coefficients $\left({ }^{48}\right)$, the mean value of the intrinsic angular momentum is :

$$
\begin{aligned}
& \langle J\rangle=l_{i}-l_{(f)}=l_{i}\left(1-\frac{y_{\text {rel }}}{y_{\text {tot }}}\right)\left[1-\exp \left(-\frac{y_{\text {tot }} t}{j \text { rel }}\right)\right] \\
& \text { related to the time by intergrating. }
\end{aligned}
$$

and $\Delta \theta$ is related to the time by intergrating.

$$
\Delta \theta=\int_{0}^{\tau} l_{f} \quad \text { dt }\left(\frac{d \theta}{d t}\right)=\int_{0}^{\tau} l_{f} d t \hbar \frac{l_{(t)}}{T_{r e l}}
$$

The deformation is introduced in the moment of inertia through the distance between centers $r(t)=R_{0}(1+\alpha t)$ and the coefficient

$$
\alpha(t)=\alpha_{0}\left(1-\exp \left(-\frac{t}{\tau_{\alpha}}\right)\right.
$$

The final kinetic energy equation

$$
E_{f}=\frac{B}{1+\alpha_{f}}+\frac{\left(\hbar l_{f}\right)^{2}}{2 \mu R_{f}^{2}}+E_{\text {rad }}^{i} \exp \left(-\frac{\tau l}{\tau_{R}}\right)
$$

is coupled to the $\Delta \theta$ equation in order to determine the interaction time for a given $\ell, \tau$, with a initial radial kinetic energy $E_{\text {rad }}$

The comparison with experimental data $\left({ }^{43}\right)$ on the energy spectra and also $Z$ distributions for the system $\left({ }^{86} \mathrm{Kr}+{ }^{166} \mathrm{Er}\right)$ leads $\left({ }^{49}\right)$ to the following average relaxation times much shorter than the estimates of Schroëder et al $\left({ }^{46}\right)$ :

$$
\tau_{R}=0.310^{-21} \mathrm{sec}, \tau_{f}=1.510^{-21} \mathrm{sec}, \tau_{\alpha}=510^{-21} \mathrm{sec} .
$$

Depending on $l_{i}$ varying between $300^{f}$ and $50, \tau l_{f}$ varies from $510^{-22}$ and $10^{-20} \mathrm{sec}$.

In a schematic example, we would like to show how the three components of the energy loss are related to the time scale, in figure 26 . The width of the $Z$ distribution increases as time evolves. On the beginning the radial dissipation fits the experimental results by itself, then tangential dissipation should be added so that the sticking is reached, and finally one needs the deformation effect to explain the largest energy losses. A number of results have been recently successfully analysed in this spirit. On the other hand simpler analysis may be done for lighter systems. One of the advantage of such light systems in the narrow

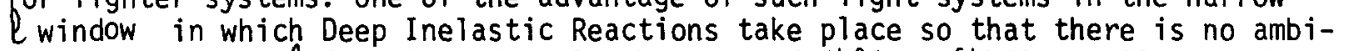
juity related to $\mathrm{l}$-fractionation. In the case of $\left({ }^{40} \mathrm{Ca}+{ }^{64} \mathrm{Ni}\right)$ at $182 \mathrm{MeV}$, the kinetic energy loss has been observed $\left({ }^{22}\right)$ from 15 to $70 \mathrm{MeV}$, in a $\mathrm{l}$-range between 58 and 67 . The c.m. angular distributions for a few selected bins of kinetic energy for the fragment $Z=18$ are shown in figure 27 after a procedure for unfolding each distribution into a positive and a negative angular contribution, since Wilcynski's plots already presented in figure 15 indicate clearly the two contri- 


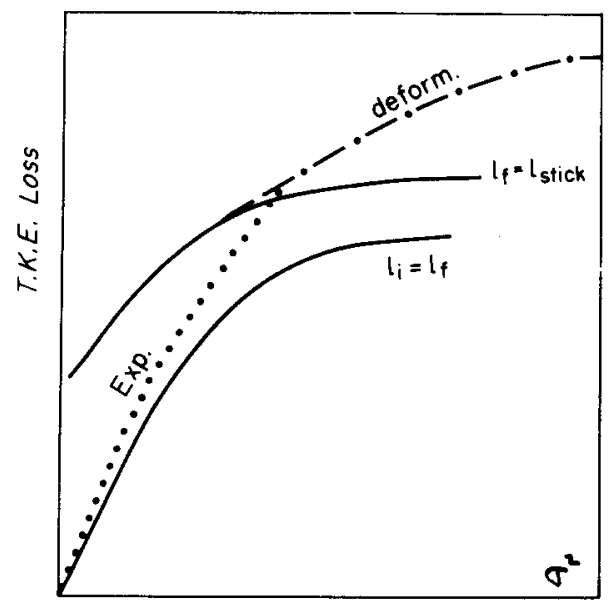

Fig. 26 : Schematic drawing of the relation between the charge width, $\sigma^{2} z$, and the radial energy dissipation $\left(\ell_{i}=\ell_{f}\right)$, the limiting tangential energy dissipation $\left(\ell_{f}=\ell_{s t i c k i n g}\right.$ ) and the influence of deformation on the moment of inertia on the total kinetic energy loss and time evolution

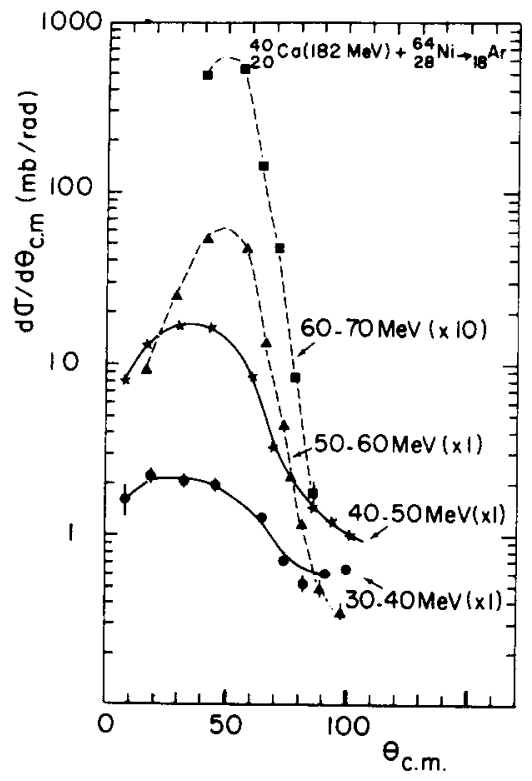

Fig. 27 : The c.m. angular distributions for selected bins in the energy spectra of $Z=18$ in the reaction $\left({ }^{40} \mathrm{Ca}+{ }^{64} \mathrm{Ni}\right)$ at $182 \mathrm{MeV}$ (ref. 22).

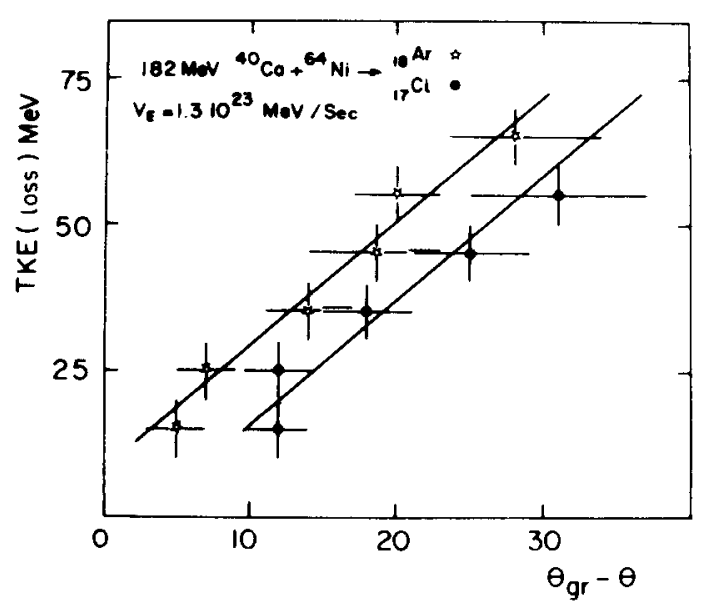

Fig. 28 : Variation of the mean total kinetic energy loss versus rotation angle of the composite system $\left({ }^{40} \mathrm{Ca}+{ }^{64} \mathrm{Ni}\right)$ for the products $Z=17$ and $Z=18$.(ref. 22 ). 
butions for $\theta<80^{\circ}$. In figure 28 , six sets of $10 \mathrm{MeV}$ bins of kinetic energy loss between 10 and $70 \mathrm{MeV}$ are shown as a function of the most probable rotation angle, for $Z=18$ and $Z=17$. Since the results can be fitted by a straight line, expressed by TKE loss = VEt, the energy drift coefficient can be deduced, and a value of $1.310^{23} \mathrm{MeV} \mathrm{sec}-1$ was found. The result is rather fast damping of the available kinetic energy (around $6 \cdot 10^{-22} \mathrm{sec}$ ). In the case of this light system, the part due to angular momentum transfer is smaller and the radial dissipation dominates. The experimental determination of $V_{E}=1.310^{23} \mathrm{MeV} \mathrm{sec}^{-1}$ is not in disagreement with the estimates made by Nörenberg $\left({ }^{5}\right)$ for the first moment of the Fokker-Planck equation applied to the energy degree of freedom

$$
\frac{\partial P}{\partial t}(E, t)=-v_{E} \frac{\partial P(E, t)}{\partial E}+D_{E} \frac{\partial^{2} P(E, t)}{\partial E^{2}}
$$

However, the microscopic derivation of the velocity coefficient gives a smaller value. In order to obtain the actual $v_{E}$, one should take a width $\Delta$ for the possible change of excitation energy in a single step as high as $4 \mathrm{MeV}$. Let us remind that $\Delta$ is related to the mean Fourier components of the time-dependent perturbation of the target nucleus :

$$
\Delta=\frac{\hbar v_{\text {rel }}}{R}
$$

where $v_{r e l}$ is relative velocity and $R$ the interaction radius. At $1 \mathrm{MeV} /$ nucleon, $\Delta$ is of the order of $2 \mathrm{MeV}$. In order to account for the large value of $V_{E}$, one could suggest again a contribution of a fast collective process for the first stage of energy damping. Also, it was found that the $Z$ distribution stays very sharp around $Z=18$ as far as the total kinetic energy loss is smaller than $30 \mathrm{MeV}$.

The width of the $Z$ distribution increases significantly only when the kinetic energy loss has reached $30 \mathrm{MeV}$. The initial stage of the energy damping occurs with a very small change in the variance of the charge distribution, so that one might again believe that the mechanism is a non-statistical one. The energy relaxation occuring in a second step follows the diffusion model like the relaxation of the $Z$ asymmetry degree of freedom.

To conclude on this subject, the radial kinetic energy damping is a rather fast process $\left(0.310^{-21} \mathrm{sec}\right)$. Then the dissipation of relative angular momentum may take more time specially for heavy system when the \& population goes very far $\left(10^{-21} \mathrm{sec}\right)$. In order to reach the lowest kinetic energy observed for the fragments, deformation has to develop during somewhat larger times. Although there is still some controversy on the subject $\left({ }^{52}\right)$, mass asymetry equilibration takes more time (up to $10^{-20} \mathrm{sec}$ ) so that the width $\Gamma_{Z}$ still increases substantially whereas the lowest kinetic energy has been reached. 


\section{FIRST STAGE IN ENERGY DISSIPATION AND THE N/Z EQUILIBRATION.-}

The preceeding studies were made on heavy systems and without a separation of each mass. The main purpose was to understand statistical aspects of the energy loss. Another approach is to try to discover which are the doorway states leading to the energy absorption. This is particularly true for the first steps of energy dissipation where it is difficult to assume that equilibrium has already been reached, or at least that the intrinsic degrees play the role of a heat bath. As a matter fact, the first stage of dissipative collisions is certainly dominated by coherent motion of nucleons in their mean single-particle potential, and later on, i.e., after $5.10^{-22}$ or $10^{-21} \mathrm{~s}$, it decays to a complex system of states via residual interactions. Therefore if one keeps the idea of energy loss due to nucifon exchange, the doorway configuration is to be searched in a non-adiabatic behaviour of nucleons, and described as a highly correlated state of particlehole excitations. However, another possibility has been proposed by Broglia et al $\left({ }^{59}\right)$. It consists in some type of rapid energy dissipation through the collective excitation of surface modes at the early stage of the collision.

In a first set of calculations, the dynamics of the surface deformation and the damping of vibrational modes were studied, using the surface-surface interaction. Recently the mass transfer between the two nuclei has been included in the treatment $\left({ }^{59}\right)$, and it seems that surface vibrations and particle transfers play equally important roles. If this is true, no doubt that the effect of nucleon exchange would wash out the structures due to the surface vibration mode in the energy dissipation.

At the present time, there is no definitive experimental answer to the question whether or not a collective dissipation mode is needed in order to explain the energy damping. In principle the time for a collective excitation is typically several time smaller than the collision time, estimated as the time necessary to travel along $R_{1}+R_{2}$ with the relative velocity $v$, so that

$$
\frac{\hbar}{\tau_{\operatorname{col} 1}}=\frac{h v}{R_{1}+R_{2}} \# 3 \mathrm{MeV}
$$

If one derives the time for collective excitation $\tau_{\text {exc }}$ from the force exerted along 1 fermi (unstead of $R_{1}+R_{2}=10 \mathrm{fm}$ ):

$$
\frac{\hbar}{\tau_{\text {exc }}}=\frac{\hbar v}{1 \mathrm{fm}}=30 \mathrm{MeV}
$$

This shows that $\tau_{\text {exc }}$ is 10 times shorter than $\tau_{\text {coll }}$.

The observation of giant quadrupole resonances in heavy ion scattering experiments has been reported $\left({ }^{54.55}\right)$. Recently, the excitation of giant multipole resonances was induced $\left({ }^{56}\right)$ by ${ }^{16} 0$ projectiles on ${ }^{12} \mathrm{C},{ }^{58} \mathrm{Ni}$ and ${ }^{208} \mathrm{~Pb}$. Highly excited modes around $20 \mathrm{MeV}$ were found in $208 \mathrm{~Pb}$. It is no doubt that striking evidence has been presented for the possibility of excitation of strongly collective modes as a doorway process for energy transfer from the relative motion to excitation of the fragments. But of course, this has been observed in inelastic scattering because the exit channel could exhibit very clearly the characteristics of energy release in the spectrum. When deep inelastic reactions occur, the collective excitation will probably decay most of the time in many intrinsic states so that no particular structure will be observed in the energy spectra of the products. Therefore it might be quite difficult to find out some direct experimental evidence of the participation of giant resonance excitation into the energy dissipation. However, there are two experimental results which may bring more confidence in the possibility of including this mechanism in the dissipation process. The first set 
of experimental data has been obtained in Orsay $\left({ }^{5}{ }^{7}\right)$ in a detailed study of the energy spectra of fragments emitted after partially relaxed collisions on $\left({ }^{40} \mathrm{Ca}+{ }^{40} \mathrm{Ca}\right)$ at $284 \mathrm{MeV}$ and $\left({ }^{63} \mathrm{Cu}+{ }^{63} \mathrm{Cu}\right)$ at $450 \mathrm{MeV}$. A more recent result has been obtained $\left({ }^{58}\right)$ on $\left({ }^{40} \mathrm{Ca}+{ }^{\circ} \mathrm{Ca}\right)$ at a higher energy of $400 \mathrm{MeV}$. Let us summarize the most interesting point. For some fragments of $Z$ and $A$ close to ${ }^{40} \mathrm{Ca}$, the energy spectra do not show a continuous descent from quasi-elastic energies down to low kinetic energies. A well marked bump appears at an excitation energy around $50 \mathrm{MeV}$, which is particularly pronounced when the spectrum has been taken for an individual nucleus ( $Z$ and $A$ identification), typically ${ }^{41} \mathrm{Ca},{ }^{42} \mathrm{SC},{ }^{36} \mathrm{Ar}$ or ${ }^{44} \mathrm{Ti}$ in the case of ${ }^{40} \mathrm{Ca}$, or ${ }^{64} \mathrm{Zn}$ in the case of ${ }^{63} \mathrm{Cu}$. These structures were observed at angles slightly inside the grazing angle, i.e., between $7^{\circ}$ and $14^{\circ}$. In addition to the $50 \mathrm{MeV}$ peak, less marked humps seem to appear at 30 and even at $85 \mathrm{MeV}$ in the last experiment $\left({ }^{\circ 0} \mathrm{Ca}\right.$ at $\left.400 \mathrm{MeV}\right)$, as shown in figure 29 . Furthermore, the $Z$ as well as the $N$ width were found still rather narrow in the range of excitation energies around $50 \mathrm{MeV}$. It means that very few particles are emitted, as expected if the statistical equilibrium is not achieved.
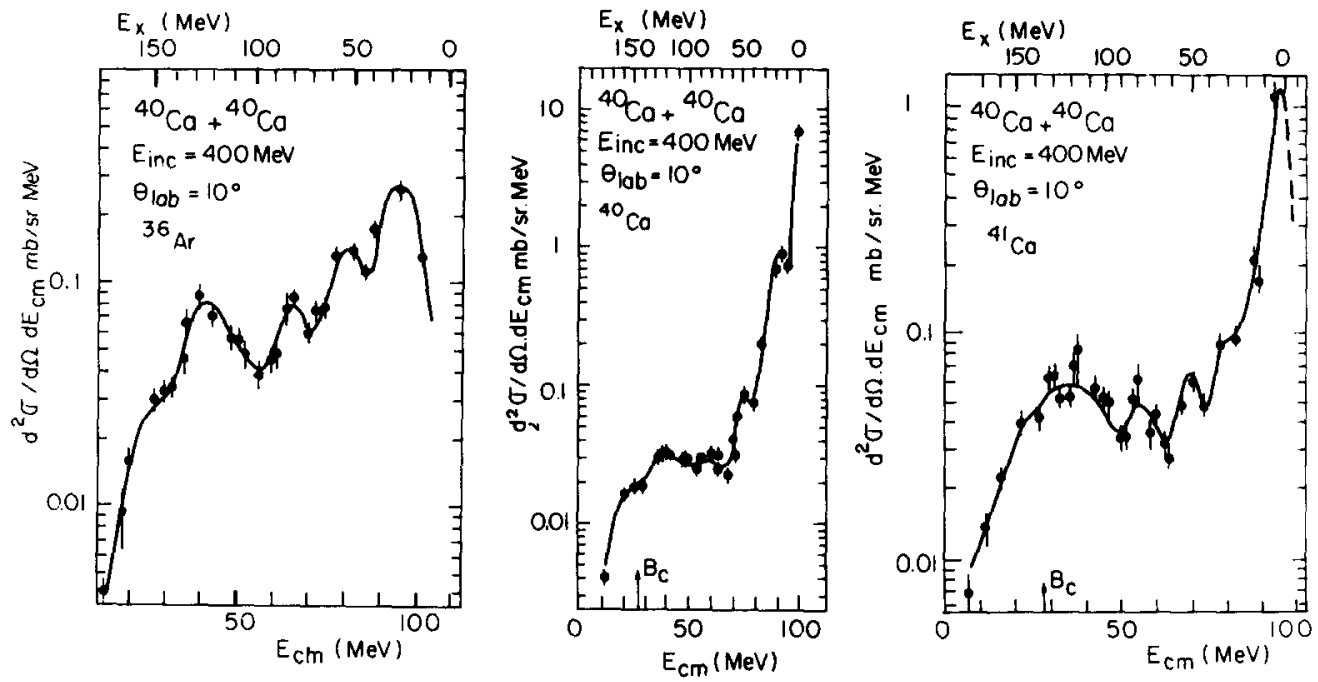

Fig. 29 : Energy spectra for several fragments close to the projectile ${ }^{40} \mathrm{Ca}$, in the deep inelastic events produced in the reaction $\left({ }^{40} \mathrm{Ca}+{ }^{40} \mathrm{Ca}\right)$ at $400 \mathrm{MeV}$. We 11 marked bumps appear at 30 and $55 \mathrm{MeV}$ (ref. 49).

It is quite tempting to interprete these results as an evidence for giant multipole resonance energy absorption. From the place in excitation energy, this would correspond to quadrupole $(15+15 \mathrm{MeV})$ and octupole $(30+30 \mathrm{MeV})$ isos-
calar modes.

However, the experimental results should be analyzed with great care, because one should be aware that when target and projectile have equal masses, the observation of events at a given angle, $\theta_{\text {obs }}$, might correspond to four disruption angles after 4 different rotation angles, $\Delta \theta$. On the classical Wilczynski's contour plots for the projectile-like fragment, the positive branch of the $\Delta=f(\bar{\theta})$ ridge corresponds to $\Delta \bar{\theta}_{1}=\bar{\theta}_{\mathrm{gr}}-\bar{\theta}_{\text {obs }}$, in the $c$ of $\mathrm{m}$ system and the negative branch corresponds to a greater rotation $\Delta \bar{\theta}_{2}=\bar{\theta}_{\text {gr }}+\bar{\theta}_{\text {obs }}$. But now the same fragment mass could also came from the target since $A_{T}=A_{p}$. Then, originated from the target grazing angle, a positive and a negative branch contributes also to the emission of fragments at $\bar{\theta}_{\text {obs }}$, but after $\Delta \bar{\theta}_{3}=180-\left(\bar{\theta}_{\text {gr }}+\bar{\theta}_{\text {obs }}\right)$ due to target "negative" $\bar{\theta}_{\text {gr. }}$. 
and after $\Delta \bar{\theta}_{4}=180+\left(\bar{\theta}_{\text {gr }}-\bar{\theta}_{\text {obs }}\right)$ due to target "positive" $\bar{\theta}_{\text {gr. }}$. For $\Delta \bar{\theta}_{4}$, which is greater than $180^{\circ}$, complete relaxation is probably achieved and this corresponds to an average excitation energy of $140 \mathrm{MeV}$. But, the bump at $\mathrm{E}^{*}=85 \mathrm{MeV}$ could be at tributed to partial energy losses selected by the rotation $\Delta \bar{\theta}_{3}$ which is of the order of $120^{\circ}$. Perhaps also the bump at $E^{*}=50 \mathrm{MeV}$ could correspond to the effect of the negative branch after rotation $\Delta \bar{\theta}_{2}$, so that the only real structure would be the bump appearing at $30 \mathrm{MeV}$ on the spectrum which decreases from the quasi-elastic scattering energy and it could be attributed to the quadrupole giant resonance of absorption effect. Moreover, a quantitative estimation has been made recentiy explaining the three bumps on the basis of simple angular effects in a symetric system $\left({ }^{93}\right)$. Therefore, the existence of real structures in the energy dissipation spectra is not yet entirely established.

\section{THE CHARGE TO MASS EQUILIBRATION AND THE ISOVECTOR GIAN DIPOLE RESONANCE}

An indirect approach in order to elucidate the role of collective degrees as doorway states for dissipative collisions is the charge equilibration. The dipole isovector giantresonance is indeed involved in the charge distribution

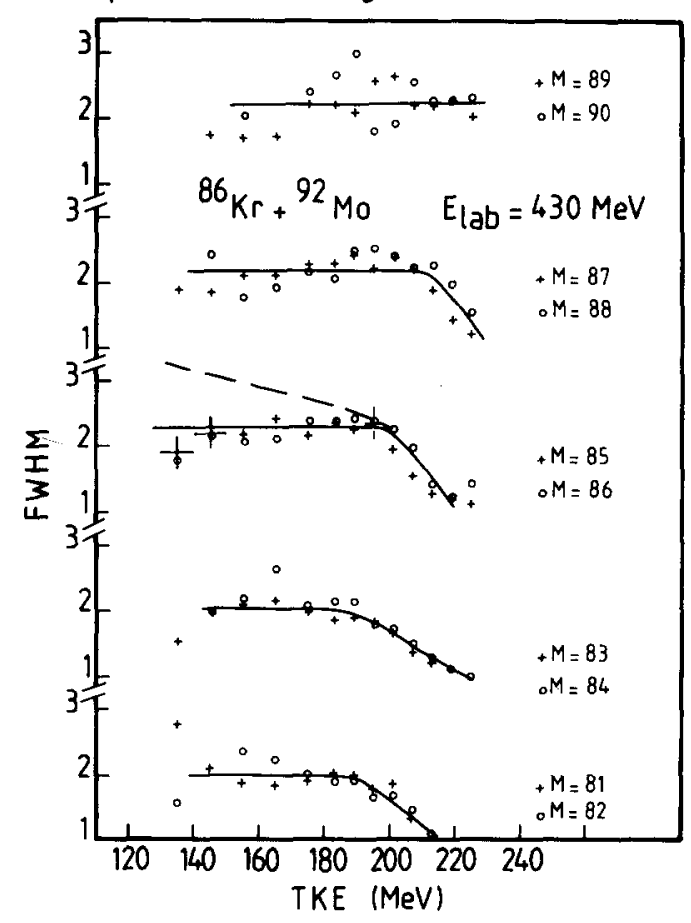

Fig. $30: Z$ distribution width for fixed mass number versus total kinetic energy loss for deep inelastic products obtained in the reaction $\left({ }^{86} \mathrm{Kr}+{ }^{92} \mathrm{Mo}\right)$. The dotted line expresses what would be a temperature depandent correlation in the case of $A=86(\Gamma \propto \sqrt{T / C})($ ref. 52).

$$
\varepsilon=E_{L D}\left(A_{1}, Z_{1}\right)+E_{L D}\left(A_{2}, Z_{2}\right)+\frac{Z_{1} Z_{2}^{2}}{R}+V_{N}(R)+\frac{\ell(\ell+1) \hbar^{2}}{2}
$$

Since $Z_{1}+Z_{2}=Z$ and $A_{1}+A_{2}=A, \varepsilon$ depends on $7 y$ on $A_{1}$ and $Z_{1}$ and for fixed $A_{1}$, 
$\varepsilon\left(Z_{1}\right)$ is a parabola since

$$
E_{L D}\left(A_{1}, Z_{1}\right)=-a_{v} A_{1}+a_{s} A_{1}^{2 / 3}+a_{c} \frac{Z_{1}^{2}}{A_{1}{ }^{1 / 3}}+\frac{1}{2} a_{s y m} \frac{\left(N_{1}-Z_{1}\right)^{2}}{A_{1}}
$$

with the usual coefficients of the mass formula $a_{y}, a_{s}, a_{f}$ and $a_{s y m}$. The minimization of the potential energy is driving the system to the most probable $\left\langle z_{1}\right\rangle$ value, $\left.\frac{d \varepsilon}{d Z_{1}}\right|_{A_{1}}=C_{0}=0$ and the most important terms are those connected to the symmetry energy. The second moment of the atomic number distribution is less sensitive that the mean value $\left\langle Z_{1}\right\rangle$ to the subsequent evaporation of particles. Now we have to understand $i$ ts physical meaning. Since the potential energy versus $z_{1}$ is a parabola, one may describe the neutron excess mode by an harmonic oscillator. But it is put in a heat bath at a temperature $T$ which corresponds to the intrinsic degrees. Then we have the usual expression relating the variance to the collective frequency $\omega$ and the temperature $T$.

$$
\begin{gathered}
\sigma_{Z Z}^{2}=\frac{1}{B \omega^{2}}\left(\frac{\hbar \omega}{2}+\frac{\hbar \omega}{\exp \left(\frac{\hbar \omega}{T}\right)-1}\right) \\
\left(B \omega^{2} \text { is the stiffness } c\right)
\end{gathered}
$$

where $B$ is the inertia. We consider two limiting cases :

i) when $\hbar \omega \ll T, \sigma_{Z Z}^{2}=T / B \omega^{2}=\frac{T}{\varepsilon}$. This is not the case since the results show that the width $\Gamma$ does not increase with the energy loss as $\sqrt{T}$.

ii) $\hbar_{\omega}>T$, so that

$$
\sigma_{Z Z}^{2}+\frac{\hbar}{2 B \omega}=\frac{\hbar \omega}{2 c}
$$

This is independent of the temperature and we observe a zero point motion (Quanta) fluctuations).

Since figure 30 shows a constant value of $\sigma_{Z Z}$ (for example at $A_{1}=86$ ), one may conclude that the phonon energy is mych greater than the temperature. Taking a stiffness coefficient $c=4.5 \mathrm{MeV} / \mathrm{Z}^{2}$, the expression (30) gives a value of $\hbar \omega$ around $8.6 \mathrm{MeV}$ which is very close to the usual value deduced for the gian $E_{1}$ resonance in a double nucleus $A_{1}+A_{2}$.

$$
\hbar \omega=\frac{79}{A_{1}{ }^{1 / 3}+A_{2}{ }^{1 / 3}}=8.8 \mathrm{MeV}
$$

Furthermore, the estimates of the time necessary for relaxing the kinetic energy is of the order of $3.10^{-22} \mathrm{sec}$, according an expression similar to relation(2) applied to the system $\mathrm{Kr}+\mathrm{Mo}$. Since the plateau for the FWHM constant value is reached after only $30 \mathrm{MeV}$ of kinetic energy loss over $100 \mathrm{MeV}$, this gives a relaxation time of the order of $10^{-22} \mathrm{~s}$ for $N / Z$ degree of freedom, very comparable to the time derived from hw in relation (31).

The conclusion of this set of experiments is that the giant dipole resonance may play a strong role in the $N / Z$ equilibration. It might also, through this intermediate, contribute to the energy dissipation, specially if the $E_{1}$ mode is coupled to the intrinsic degrees of freedom.

Unfortunatly a few other results do not lead to the same clear-cut result. For the reaction ( $\mathrm{Ar}+\mathrm{Ni}$ ) at $280 \mathrm{MeV}$, mass and charge distributions have 
been studied in detail $\left({ }^{62}\right)$. The value of $\sigma z^{2}$ which can be deduced from $\hbar \omega$ according(31) is of the order of 0.7 charge units) ${ }^{2}$, whereas the measured $Z$ distribution at constant $A$ was only $\sigma z^{2}=0.3$ (ch.u.) ${ }^{2}$. This value was reprgduced with a classical statistical temperature dependent distribution, namely $\sigma \mathrm{Z}^{2}=\mathrm{T} / \mathrm{c}$.

However, in a very recent paper, Moretto $\left({ }^{63}\right)$ has suggested that $\sigma z_{2}^{2}$ might decrease strongly when $E$ increases. Furthermore the value of 0.3 (c.u. $)^{2}$ could still be associated with a dipole giant resgnance if one considers that the double nucleus is not indeed represented by $\left(A_{1} 1 / 3+A_{2} 1 / 3\right)$ in equation (31). As a matter fact it is known in fission studies, that the neck increases the inertia and the value of $h_{\omega}$ is probably much smaller than $10 \mathrm{MeV}$. A value around $4 \mathrm{MeV}$ is more reasonable in this asymmetric system and would fit with the measured width $\sigma_{Z}^{2}=0.3$ (c.u. $)^{2}$.

More recentiy, A distributions for fixed $z$ were measured $\left({ }^{64}\right)$ for $Z=12$ to $Z=17$ in the reaction $\left({ }^{40} \mathrm{Ar}+2{ }^{38} \mathrm{U}\right)$ at $263 \mathrm{MeV}$ and for $Z=10$, to $Z=14$ in the reaction $\left({ }^{32} \mathrm{~S}+{ }^{2{ }^{3}} \mathrm{U}\right)$ at $243 \mathrm{MeV}$. The authors $\left({ }^{64}\right)$ were able to account for the measured width only with statistical asumptions, i.e., thermal fluctuations resulting from one-body energy dissipation. The model starts again with a potential energy at the scission point calculated on the basis of the liquid drop formula. But in this parabolic potential curve, when plotted versus asymmetry at fixed $Z$, it was assumed a thermal excitation energy with a temperature given by $E^{*}=A^{A} T^{2}$ and a probability for producting a nucleus $(Z)$ when $A_{1}$ is fixed obtained by the Boltzmann distribution

$$
P\left(Z, A_{1}\right) \propto \exp \left(-\frac{V\left(Z, A_{1}\right)}{T}\right)
$$

where $V\left(Z, A_{1}\right)$ is the $Z$ dependent potential energy. Now the probability to obtain mass $A_{1}$ when the projectile has a mass $A_{p}$, was computed according the transport theory of Nörenberg.

$$
P\left(A_{1}\right)=\left(4 \pi D_{A} t_{R}\right)^{1 / 2} \exp \left(-\frac{A_{1}-A_{p}-v t_{r}}{4 D_{A} t_{R}}\right)
$$

with the usual drift and diffusion coefficients for mass transfer and $t_{R}$ the reaction time.

Then the probability for producting nucleus $\left(Z_{1}, A_{1}\right)$ is obtained through the normalized product $P\left(Z, A_{1}\right) \cdot P\left(A_{1}\right)$. The diffusion coefficient was calculated according Norenberg's data $\left({ }^{65}\right)$. Furthermore, one has to make a correction for neutron evaporation from these neutron rich fragments, in order to deduced from the experimental distributions the distribution prior neutron emission. Finally, the results are shown in figure 31 . The striking conclusion is indeed that the $N / Z$ degree of freedom seems to follow statistical fluctuations with a variance typically expressed by thermal effects. This would imply that either the giant resonance does not contribute strongly to the process, or some additional effect conceals its observation. 

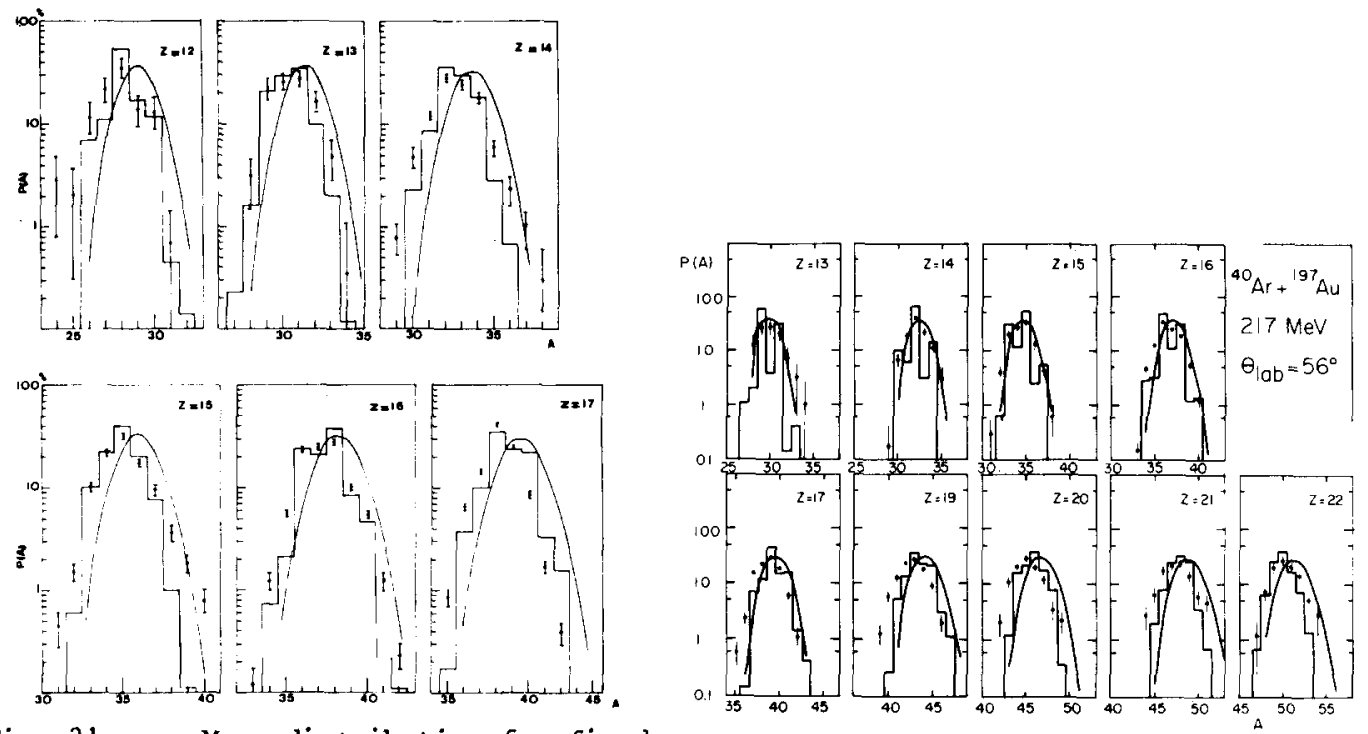

Fig. 31.a : Mass distribution for fixed values in the products of the reaction $\left({ }^{40} \mathrm{Ar}+238 \mathrm{U}\right)$. The continuous curve represents the calculation according equations (32) and (33). The histogramm is obtained after correcting for neutron evaporation (ref. 54).

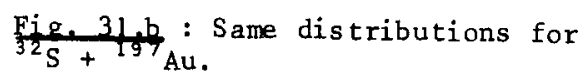

\section{DEGREE OF THERMALIZATION OF THE DISSIPATED ENERGY. SHARING OF EXCITATION ENERGY BETWEEN THE FRAGMENTS.}

All models based on statistical asumptions for explaining the main features of dissipative collisions prescribe implicitely that a statistical equilibrium is obtained for the intrinsic degrees of the fragments. If so, the excitation energy should be shared between the two fragments in proportion to their masses, and the de-excitation processes should show up all the total amount of energy which has been dissipated. That means that light particles and gamma rays should exhibit the usual behaviour observed in the evaporation mechanism.

Several sets of experiments have shown that, to a large extent, the dissipated kinetic energy is indeed thermalized in both fragments which afterwards evaporate light particles. However, in some cases, a small fraction of emitted light charged particles cannot be attributed to a normal evaporation mechanism. Their angular distribution is strongly focused towards a wall defined direction so they are not emitted by the recoiling fragments.

In a first part we shall consider the results relative to the dominant feature, $i . e .$, the statistical decay of the excited fragments.

\subsection{Fragment de-excitation by classical evaporation process}

The first approach is an indirect way resulting actually from the check of the binary nature of Deep Inelastic Reactions. By detecting in coincidence at given correlation angles both fragments and measuring their kinetic energies and velocities. One can reconstitute the kinematics. Secondary masses of the two fragments are obtained by the $E$ and $v$ measurements. Average primary masses can be deduced from the kine-matics since the correlation angle and the energies are known. 
The difference between primary and secondary masses gives the number of emitted nucleons.

A simple procedure is to identify in coincidence $Z_{1}$ and $Z_{2}$. The mean missing charge is $\Delta Z=Z_{p}+Z_{T}-\left(Z_{1}+Z_{2}\right)$, where $Z_{R}$ and $Z_{T}$ are the atomic numbers

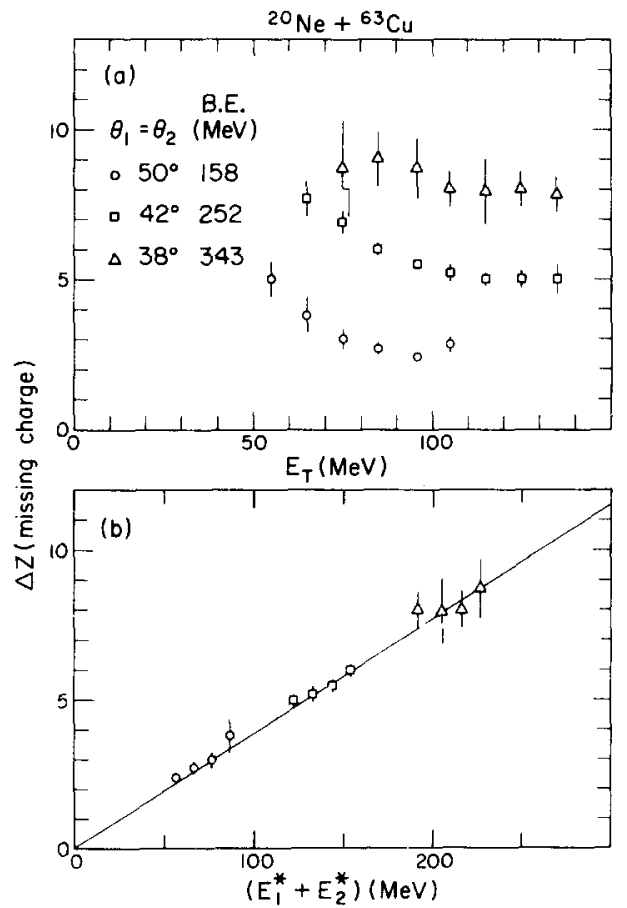

Fig. 32 : a- Mean missing charge versus $\mathbf{E}_{\mathrm{T}}$ for three different bombarding energies.

b- Number of charges missing after symmetric splitting versus excitation energy in the reaction $\left({ }^{20} \mathrm{Ne}+{ }^{63} \mathrm{Cu}\right)$ at three bombarding energies (ref. 66). of projectile and target respectively. Schmitt et al( $\left.{ }^{66}\right)$ have applied this method to the system ${ }^{20} \mathrm{Ne}+{ }^{63} \mathrm{Cu}$ at $7.9,12.6$ and $17.2 \mathrm{MeV} /$ nucleon and for different various kinetic energy cuts of nearly equal $z$ fragments. The choice of the correlation angle was made on the assumption of symmetric exit channe1. Figure 32 shows that $\Delta Z$, the number of charges emitted increases, when the kinetic energy of the fragments decreases, and when this is transformed into excitation energy, it increases linearly with $E_{1}{ }^{*}+E_{2}{ }^{*}$ with a slope of $25 \mathrm{MeV} /$ charge. Assuming that $\triangle A=2 \Delta Z$, the result of an average of $12.5 \mathrm{MeV}$ per nucleon is consistent with the evaporation model.

However, one cannot exclude preequilibrium emission and the result gives on ly a broad conclusion that there is no very big discrepancy between the behaviour of the fragment kinematics and the concept of a uniform temperature for the composite system.

More sophisticated experiments were made on the systems in which not only atomic numbers but masses were identified, and therefore a11 kinematic quatities were derived.

The mass of the light fragment emitted prior particle emission is deduced from momentum conservation :

$$
m_{L}=\frac{m_{0} v_{0}}{v_{L}} \frac{\sin \theta_{H}}{\sin \left(\theta_{H}+\theta_{L}\right)}
$$

where $m_{0} v_{0}$ is the initial momentum and $\theta_{H}, \theta_{L}$ the emission angle for heavy and light fragments, respectively.

The measured mass is obtained after particle emission

$$
\mu_{L}=2 E_{L} / V_{L}^{2}
$$

The difference $m_{L}-\mu_{L}=v$ is the number of emitted nucleons. 
If one wants to know what sort of particles are emitted, the determination of atomic numbers of both fragments is necessary so that the total charge deficit in the exit channel could be deduced. Moreover, the width of the fragment angular correlation should be analysed, since the contribution to the angular distribution variance expected from recoils effects after evaporation depends strongly on the mass of the particle evaporated. More precisely, when a particle $\nu$ of mass $\mathrm{m}_{v}$ is emitted with energy $\varepsilon_{\nu}$ (in the fragment c.m.) and when the fragment has a c.m. primary kinetic energy $E_{L}$, then the variance is given to the first order by

$$
\Delta \sigma_{\theta}^{2}=\frac{1}{2} m_{L} \frac{m_{\nu}}{E_{L}} \cdot \frac{\varepsilon_{\nu}}{\left(m_{L}-m_{v}\right)^{2}}
$$

Due to difficulties in detecting accuratly low energy heavy fragments, experiments of this type have been restricted to light or medium mass systems such as $(S+T i)\left({ }^{67}\right),(A r+N i)\left({ }^{68}\right)$ and $(A r+A g)\left({ }^{69}\right)$. Even so, the exact mass after evaporation $m_{L}$ was measured only up to $A=50$, so that the heavy fragment mass was not accuratly determined.

If there was no charged particle evaporation, it would be possible to obtain the charge and the preevaporation mass of the complementary fragment by simple difference with the composite system. But if one does so, a discontinuity appears at symmetry, as shown on the system ( $\mathrm{Ar}+\mathrm{Ni})$ studied at Orsay $\left({ }^{68}\right)$ or on the system $(A r+A g)$ studied at Berkeley $\left({ }^{69}\right)$. The gap between the two straight lines on the diagram of figure 33 represents twice the number of charges evaporated by the light fragment at symmetric mass splitting. In the case of $\mathrm{Ar}+\mathrm{Ni}$, the symmetric splitting corresponds to $\frac{1}{2}\left(Z_{1}+Z_{2}\right)-2$. In the case of $\mathrm{Ar}+\mathrm{Ag}$, Moretto et al, have found a shift of $1.3^{2}$ charge unit per fragment. Evaporation calculations predict 1.28 evaporated charges at symmetry. Then, with this correction, figure 34 indicates very nicely a linear relationship between $v$ and the $Z$ of the fragment.

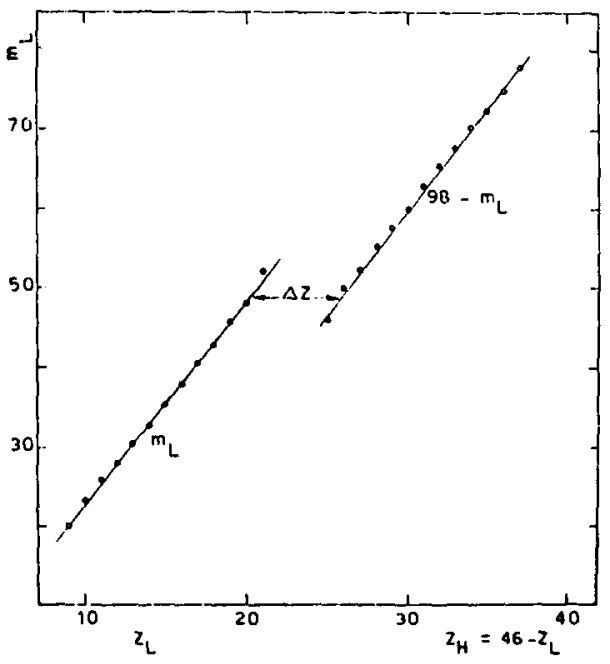

Fig. 33 : - System ${ }^{40} \mathrm{Ar}+{ }^{58} \mathrm{Ni}$. Two-body coincidence measurements 67 .

- Variation of the pre-evaporation mass $\mathrm{mL}_{\mathrm{L}}$ of the light fragment as a function of the measured charge $\mathrm{Z}_{\mathrm{L}}$. On the right hand side are plotted the results for complementary heavy fragments assuming $\mathrm{m}_{\mathrm{H}}=98-\mathrm{m}_{\mathrm{L}}$ and $\mathrm{Z}_{\mathrm{H}}=46-\mathrm{Z}_{\mathrm{I}}$.

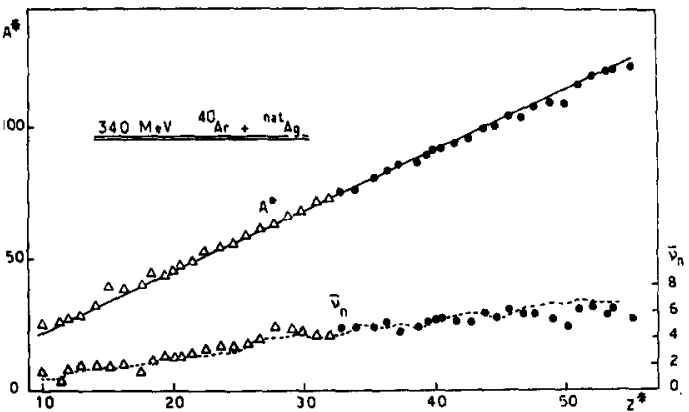

Fig. 34 : Number of missing mass units, $\bar{v}_{n}$, versus $Z$ of the fragments in deep inelastic reactions induced by ${ }^{40} \mathrm{Ar}$ on $\mathrm{Ag}$ tar gets, deduced by difference between measured masses and the mass of the composite system (after correction of 1.3 charge units) (ref, 69). 
Also inclusive measurements $\left({ }^{70}\right)$ of the fragment charges in a system which has been choosen symmetric in the entrance channe $1,{ }^{40} \mathrm{Ca}+{ }^{40} \mathrm{Ca}$, give the result of $\Delta Z=2$, quite consistent with the preceding data since the system is less neutron rich and favors charged particle emission. Finally a beautiful illustration of the observation $\left({ }^{52}\right)$ of the results of the amount of neutron emission increasing when the total kinetic energy of the fragments decreases is indicated in figure 35 where contour plots are shown for the number of events versus TKE and masses at fixed $Z$ values of the products in the reaction ${ }^{86} \mathrm{Kr}+{ }^{92} \mathrm{Mo}$.

However, the limitation of the methods described above is that they give no information on the particle kinetic energy spectra and they cannot exclude preequilibrium emission by the fragments. A few experiments have been carried on to measure light particles emitted in coincidence with the fragments. For the moment the study of neutron emission in the case of rather heavy fragments has been the more extensive work. Because of the absence of Coulomb field, neutron velocities in the frame of the emitting fragment are lower than those of charged particles and therefore the composition of neutron velocity and emitting nucleus velocity results into a cone of velocity vectors which can be identified rather unambiuously. It is possible to know by simple kinematical treatment from which fragment neutrons have been emitted as shown in figure 36 . On the other hand, neutron kinetic energy measurements are not very easy, since the scattering on any

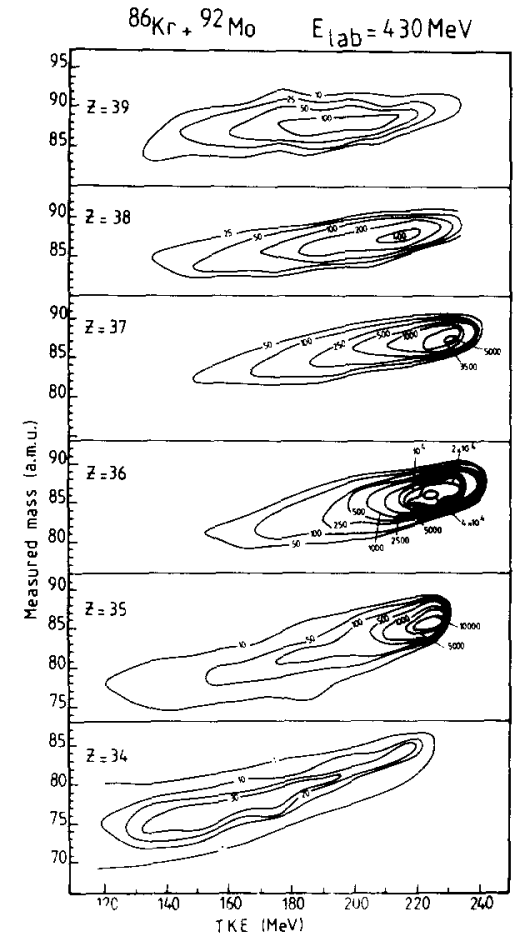

Fig. 35 : Contour plots of the differential cross section $d^{3} \sigma / d E d A$ as a function of total kinetic energy and mass number for several $Z$ values of fragments in the reaction $\left({ }^{86} \mathrm{Kr}+{ }^{92} \mathrm{Mo}\right)$. When the kinetic energy loss increases, the peak of the contour plot shifts to lower A values, which corresponds to a greater number of evaporated neutrons (ref. 52).

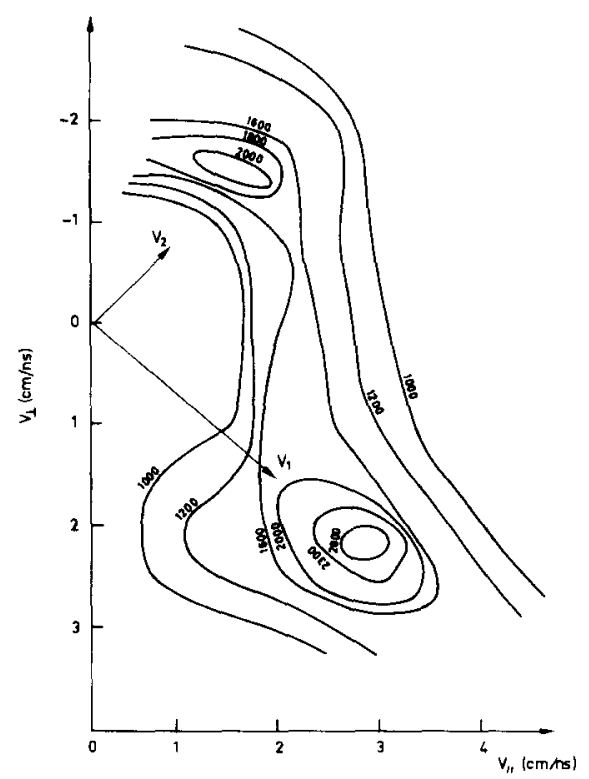

Fig. 36 : Contour plot $d^{2} \sigma / d v_{1} \cdot d v_{/ \prime}$ for neutrons in coincidence wi th fragments, in the reaction ${ }^{63} \mathrm{Cu}+{ }^{197} \mathrm{Au}$. $v_{\prime \prime}$ and $v_{+}$are the components of the neutron velocities para1lel and perpendicular to the beam direction respectively (ref. 72). 
material can modify the time of flight spectrum. There are a few sets of experimental data on systems where the fragments deexcite only by neutron emission and $\gamma$ rays.

The first one deals( $\left.{ }^{71-72}\right)$ with neutron and gamma multiplicities and neutron energy spectra from various components of the deep inelastic products in

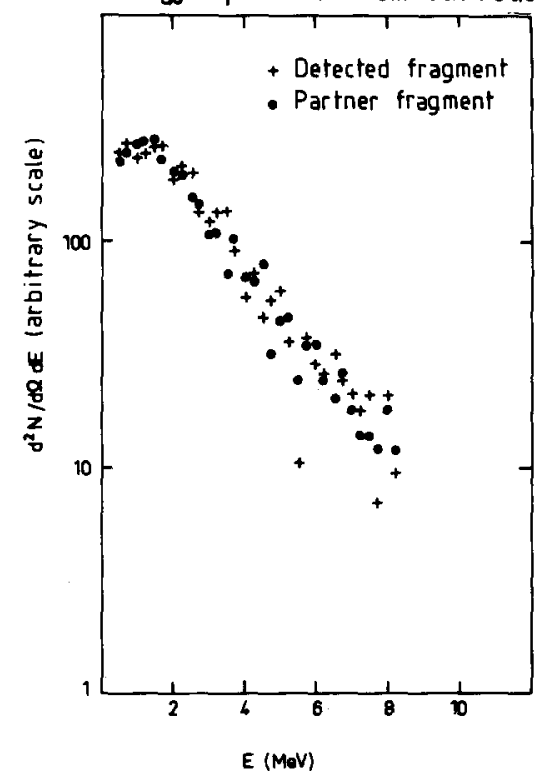

Fig. 37 : Center of mass kinetic energy spectra of the detected neutrons associated to the detected fragment and its partner. System $\left({ }^{63} \mathrm{Cu}+197 \mathrm{Au}\right)$ at a bombarding energy of $400 \mathrm{MeV}$ (ref. 72).

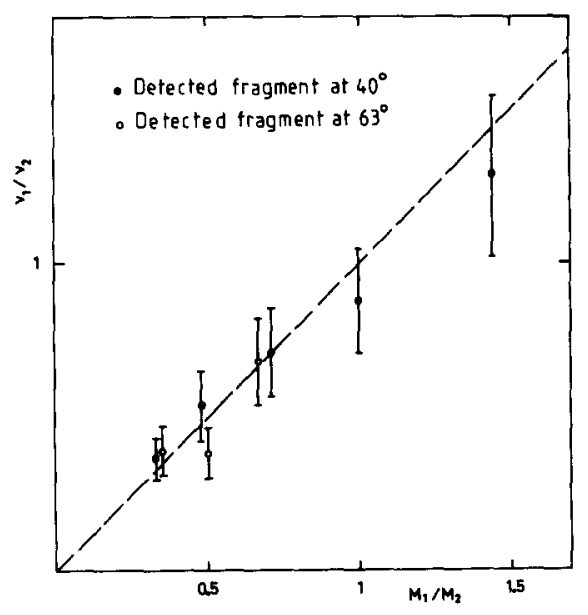

Fig. 38 : Ratio $\nu_{1} / \nu_{2}$ of the number of neutrons associated with each fragment 1 and 2 , versus their mass ratio $M_{1} / M_{2}$. the system ${ }^{63} \mathrm{Cu}+{ }^{19}{ }^{7} \mathrm{Au}$ at 365 and $443 \mathrm{MeV}$. An example of the energy spectra obtained for the detected neutrons associated to the detected fragment and its heavy partner is shown in figure 37 . It demons trates how it is connected with an evaporation behaviour. Actually a comparison was made with an evaporation code $\left({ }^{72}\right)$. Moreover, the ratio $v_{1} / v_{2}$ between multiplicities of neutrons issued from fragment 1 and the complementary fragment 2 was found proportional to the mass ratios $M_{1} / M_{2}$ (figure 38 ). This indicates that the excitation energy is shared between the two fragments in proportion to their masses, unlike in low energy fission where $\nu_{1} / \nu_{2}$ is even the inverse of $M_{1} / M_{2}$ for nearty symmetric fission where $M_{1}$ becomes close to $M_{2}$. Finally, figure 39 shows the total neutron multiplicity $\nu T$ as a function of the excitation energy ( $k$ inetic energy loss $+Q$ value). The intersect with the abscissa indicates the mean energy removed by $\gamma$

.

1Detected fragment at $40^{\circ}$ - Fragment at $63^{\circ}$

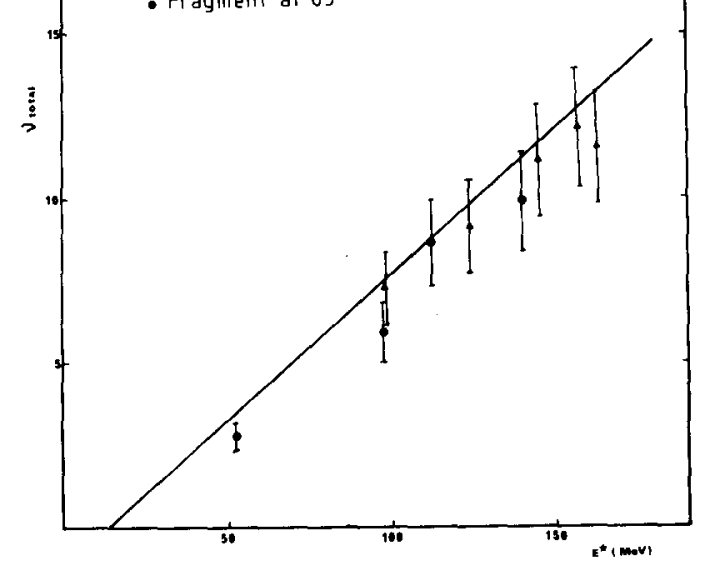

Fig. 39 : Total neutron multiplicity $\nu_{T}$ as a function of the excitation energy of both fragments. The line is obtained in a calculation assuming statistical theory. For $\nu_{\mathrm{T}}=0$ the excitation energy is due to gamma ray emission (ref. 72 ). 
rays. It is of the order of $14 \mathrm{MeV}$, and this corresponds to experimental measurements.

Even at rather low energy losses, i.e., around $30 \mathrm{MeV}$, the ratio $\nu_{1} / \nu_{2}$ is still equal to $M_{1} / M_{2}$. This means a rather fast process for equilibrating the excitation amongst the two interaction nuclei.

These results seem to prove that the assumption of statistical equilibrium among the intrinsic degrees is quite valid. Furthermore no additional component can be seen and processes like Fermi jet or hot spot emission appear probably significantly only at higher energies.

In the systems $\mathrm{Xe}+\mathrm{Au}$ at $6 \mathrm{MeV} / \mathrm{n}\left({ }^{73}\right)$ and ${ }^{56} \mathrm{Fe}+{ }^{16}{ }^{5} \mathrm{Ho}$ at $8.5 \mathrm{MeV} / \mathrm{n}\left({ }^{9}{ }^{4}\right)$, the same conclusion was reached although the total multiplicity was a bit smaller than expected. However, the results of Eyal et al $\left({ }^{74}\right)$ on $(K r+E r)$ support completely statistical models. The linearity observed between $\nu$ and the mass of the fragment shows that in both fragments the deformation energy is roughly the same, at variance with what is found in fission process. This is quite understandable, since the neck between the projectile and the target has a short life time, whereas in fission the delay between the saddle point and the scission point is rather long and scission can occur at different points of the neck so that the light fragment may be strongly deformed.

Very recentiy, a slightly different result has been obtained( $\left.{ }^{92}\right)$ in the case of $\mathrm{Ar}+\mathrm{Au}$ at $220 \mathrm{MeV}$, an energy rather close to the barrier. Because of the low kinetic energy in the entrance channel, the excitation energy is not very large, and for the lightest fragment, around $A=30-40$, the number of emitted neutrons is larger than expected from a pure statistical sharing of the energy. The difference is not very great but significative, since instead of $\nu=1.5$, a value of 2.2 was obtained. This effect might not have been seen in other cases because of the higher energy, so that the deformation energy was relatively too small. It seems therefore that there is a small fraction of the energy dissipation which remains into the surface vibration modes and leads to deformation of the shape rather than to intrinsic states. However, it is by no means equal to the part of energy dissipation which goes through nucleon exchange or particle-hole excitations, like it was predicted by Broglia et al $\left({ }^{59}\right)$. The results on neutron emission indicate very clearly that, although collective mode may play an important role, they decay into intrinsic states before the scission into two fragments, at variance with Broglia's predictions.

The detection of neutrons is probably the best way to look at the statistical de-excitation process, particularly for heavy fragments since neutron emission is the main de-excitation channel. At the inverse the detection of charged light particle will focuse the attention on reaction mechanisms which differ from the statistical evaporation, even if the amount of such processes corresponds to a small fraction of the possibilities of particle emission at any stage of the dissipative reaction. There have been several attempts for measuring protons and alpha particles emitted in coincidence with heavy fragments. In most of the cases, and particularly at the highest relative velocities, two contributions have been recognized. One which can be attributed to a statistical decay in the rest frame of the heavy recoiling fragment as well as the light fragment. A second contribution the origin of which is not clearly understood, al though it is probably related to an earlier stage of the deep inelastic collision and to the first step of kinetic energy loss.

In the system $\left({ }^{40} \mathrm{Ar}+{ }^{58} \mathrm{Ni}\right)$ at $280 \mathrm{MeV}$, both radial and azimuthal angular distributions have been measured $\left({ }^{35}\right)$ and the study of $\alpha$-particle emission is particularly interesting. For very asymmetric splittings, where the analys is is the clearest, the contribution for particles evaporated by the light fragment is restricted to a narrow cone in the laboratory system. The analysis of particles 
emitted outside this cone has shown that the angular distribution do/d $\Omega$ is flat and the kinetic energy spectrum corresponds to a temperature around $2.5 \mathrm{MeV}$ when the transformation is made into the heavy fragment rest frame. The estimated temperature of the composite system $T=\sqrt{\frac{8 E x}{A}}$ can be estimated around $2.7 \mathrm{MeV}$ which is in good agreement with the measured $A$ slope of the energy spectrum. Moreover, the azimuthal angular distribution exhibits the usual symmetry around $\phi=90^{\circ}$ (where $\phi=0$ for a direction in the reaction plane).

It is known that, according Ericson and Strutinsky $\left({ }^{76}\right)$, the out-of plane distribution depends on the angular momentum of the emitting nucleus, $J$, through an expression

$$
w_{J}(\varphi) \propto \exp \left(\frac{\hbar^{2}(J+1 / 2)^{2}}{2 J T}+\frac{m R^{2}}{J+m R^{2}} \cos ^{2} \varphi\right)
$$

where $y$ is the moment of inertia of the emitting nucleus, $m$ the mass of the $\alpha$ particle. The quantity $\mathrm{mR}^{2}$ is usually small with respect to $y$, and a popular simplified expression is often taken.

$$
W_{j}(\varphi) \propto \exp \left(\frac{E_{\text {rot }} m R^{2}}{2 T j} \cos ^{2} \varphi\right)
$$

The exponent is the ratio of the centrifugal energy of $\alpha$-particles emitted by a rotating emitter to the temperature. It can also be expressed with the usual spin cut off factor $\sigma^{2}=\frac{\pi T}{\hbar^{2}}$ and the orbi-
tal angular momentum of the particle,

$$
\frac{E_{\text {rot }} \ell^{2}}{2 \sigma^{2}}
$$

A rough estimate for the actual angular distribution has given an average value $\langle J\rangle=30 \hbar$, which fits rather nicely the angular momentum transferred to the heavy fragment in sticking conditions from an orbital angular momentum in the entrance channel equal to $85 \pi$.

The conclusion for this asymetric splitting is that all the characteristics of the statistical theory are fulfilled. However, this is not the case for more symetric splitting and a contribution appears particularly at backward angles for $\alpha$-particles in coincidence with fragments of $Z=21-23$, which exhibits higher energy spectra and do not obey the kinematics expected for an evaporation process.

\subsection{Emission of light particles at earlier stages of the reaction.}

The emission of fast $\alpha$-particles from the region of initial impact along the direction of the classical coulomb trajectories of the incoming projectile has been rather clearly observed( $\left.{ }^{77}\right)$ in the case of ${ }^{16} 0$ projectiles on $208 \mathrm{~Pb}$ and ${ }^{197} \mathrm{Au}$ at high energies (around $9 \mathrm{MeV} / \mathrm{A}$ and $18 \mathrm{MeV} / \mathrm{A}$ ). The ejectiles were identified as ${ }^{12} \mathrm{C}$ and ${ }^{13} \mathrm{C}$ and the angular dependence had shown that $\alpha$-particles in coincidence do not originate from a sequential decay of the ejectile or in a particle emission from the target like fragment. These $\alpha$-particles could be associated with the tangential friction forces and appear cor partially damped as well as for fully damped collisions. The same type of explanation has been put forward by Inamura et a $1\left({ }^{78}\right)$, when they observed a high energy forward peaked $\alpha$-component associated with large $\ell$-waves above $\ell \mathrm{cr}$ or in the reaction of ${ }^{14} \mathrm{~N}$ on ${ }^{159} \mathrm{~Tb}$. In that peculiar case, while the $\alpha$-particles were emitted, the residual part of the projectiles ${ }^{10} B$, were absorbed by the target and led to a fusion excited nucleus, ${ }^{169} \mathrm{Yb}$, which decayed by neutron and gamma emission. 
At lower energies, the process of sequential $\alpha$-particle-like transfer has been rather well established $\left({ }^{99-80-81}\right)$ in the case of $\left({ }^{14} \mathrm{~N}+{ }^{9} \mathrm{Nb}\right)$, ${ }^{27} \mathrm{Al}\left({ }^{16} \mathrm{O},{ }^{12} \mathrm{Ca}\right)^{27} \mathrm{Al},{ }^{58} \mathrm{Ni}\left({ }^{16} \mathrm{O},{ }^{12} \mathrm{Ca}\right){ }^{58} \mathrm{Ni}$ and ${ }^{58} \mathrm{Ni}\left({ }^{16} 0,{ }^{16} \mathrm{OO}\right){ }^{54} \mathrm{Fe}$. This was shown mainly by the observation that the average $Q$ value for the formation of an intermediate system is independent of the detection angle of the decay particle. However, the intermediate, for example ${ }^{31 P}$ in the ${ }^{27} \mathrm{Al}+{ }^{16} 0$ reaction $\left({ }^{80}\right)$, or ${ }^{62} \mathrm{Zn}$ in the ${ }^{5} \mathrm{Ni}+{ }_{16} 0$ reaction $\left({ }^{81}\right)$ emits $\alpha$-particles in times shorter than the one associated with statistical equilibrium. The angular distribution in the rest frame of the emitting system is not isotropic, as it should be in a thermally equilibrated nucleus, and a particle spectra exhibit a high energy tail, particularly at the maximum of the angular distribution. This is typical of a fast sequential process with only partial equilibration. But we don't know wether the emission originates just after scission of the composite system from a high temperature locus, or in a statistical decay occuring prior equilibrium as described by $B 1$ ann $\left({ }^{82}\right)$ in the case of highly excited compound nuclei.

For heavier projectiles, like S, Ar, Cu or Kr, we already know that the statistical de-excitation process produces the major fraction of the emitted light particles which are mainly neutrons. However, the study of the emission of alpha particles and protons has been recently carried out. When the energy damping is very large, the sequential character of the light particle emission is not so easily proved. For example, for the ${ }^{86} \mathrm{Kr}+{ }^{197} \mathrm{Au}$ system, Miller et al $\left({ }^{83}\right)$ have analysed light particles $(\alpha, d, p, t)$ in coincidence with the projectile-like fragments. Moreover, they have independently studied $\left({ }^{84}\right)$ the de-excitation process of a compound nucleus, ${ }^{75} \mathrm{Br}$, produced in a similar range of excitation energy and angular momentum. From the data obtained on $\Gamma_{\alpha} / \Gamma_{p}$, on energy spectra, etc..., they made a kinematic model for the de-excitation of the projectile-like fragment and they were able to originate a well defined fraction of light particle emission from the target-like fragment, based on the study of the decay of ${ }^{194} \mathrm{Hg}$ compound nuclei( $\left.{ }^{83}\right)$.

However, a third contribution of $\alpha$-particles was isolated with an angular distribution focused in the region normal to the direction of emission of the fragments in the c.m. reference frame. Also the mean kinetic energy for these $\alpha$ particles was around the coulomb barrier of the composite system, so that a possible origin could be the neck at scission. This phenomenon is rather seldom in spontaneous fission and the coulomb field of the two fragments constrains the $\alpha$ particles to be emitted in a narrow cone perpendicularly to the fragment separation line. In the case of $\mathrm{Kr}+\mathrm{Au}$ at $720 \mathrm{MeV}$, it corresponds to $0.2 \alpha$-particle per deep inelastic collision.

There are also some peculiar cases, where a-particles were measured in coincidence with fragments but for a rather small energy loss, in such conditions that perhaps specific nuclear structures may have a role. This is the case of a recent study of the system ${ }^{32} \mathrm{~S}+{ }^{19}{ }^{7} \mathrm{Au}$ at $373 \mathrm{MeV}\left({ }^{85}\right)$. A large coincidence rate was measured for $\mathrm{S} i+\alpha$, when $S i$ fragments still carry a large kinetic energy. Moreover, a strong focusing effect appeared in the angular distribution at an intermediate angle between the fragment direction and the beam direction. This would be consistent with a break-up of the excited projectile just after the collision. Perhaps the most interesting data have been obtained( $\left.{ }^{86}\right)$ recently on the system $(A r+A u)$ at a rather high bombarding energy of $9 \mathrm{MeV} / \mathrm{A}$, because they demonstrate a "fast" emission of protons and $\alpha$-particles from the composite system prior the scissions into two fragments. These emissions provide a tool for investigating the various aspects of energy dissipation in the intermediate dinucleus system.

When heavy target nuclei are bombarded by Argon ions, a large part of the reaction cross section goes into the production of fragments which presents all the characteristics of fission al though the liquid drop fission barrier is supposed to vanish. These fission-like products have a $Z$ distribution around symmetric splitting, and one angular distribution following $1 / \mathrm{sin} \theta$. However, the 
reaction occurs probably quickly and this is why Alexander et al $\left({ }^{86}\right)$ have studied protons and ${ }^{4} \mathrm{He}$ emitted in coincidence with those fission-like fragments $(Z>27)$. They found an isotropic component from the analysis of the data at backward angles, and a forward peak which is more energetic. There are several observations that indicate that both emissions preceed fragment acceleration and are originated from an earlier stage of the composite system life. The most interesting data is the peak energy of the emitted alpha particles. It is located, event at back angles at a higher value that the maximum of $\alpha$-particles emitted by the compound nucleus ${ }_{19}^{4} \mathrm{Hg}$ formed in the reaction ${ }^{12} \mathrm{C}+{ }^{182} \mathrm{~W}$ and excited at $98 \mathrm{MeV}$. It is consistent with the $Z$ ratio of $97 / 80$, so that the predominant fraction of $\alpha$-particle emission (as we 11 as proton emission) originates from ${ }^{237} \mathrm{BK}$ rather than from ${ }^{197} \mathrm{Au}$. This emission preceeds a fission-like break-up in a composite system which has a short life-time but nevertheless where complete energy damping has occured since the fragments have typically coulomb repulsion energies, and furthermore the memory of the entrance channel has been erased by a complex mass and charge exchange.

But for the backward angles, such an emission exhibits energy spectra which fit a nuclear temperature of $2.4 \mathrm{MeV}$, very close so that observed for excited heavy nuclei, i.e., it is consistent with a completely equilibrated compound nucleus, al though we know that the compound nucleus ${ }^{23}{ }^{7} \mathrm{Bk}$, if ever formed, has a dominant probability for fission and a very low chance for charged particle emission ; since the fission barrier is only $2 \mathrm{MeV}$ for $\mathrm{J}=0$ and is zero for $\mathrm{J}=10$. The conclusion is clearly the non-participation of fission channels in the particular equilibrium model which should be applied to the composite system $\left({ }^{40} \mathrm{Ar}+197 \mathrm{Au}\right)$.

The forward-peaked component is also associated to the fission like break-up. The energy spectra exhibits a high temperature $(T=3-7 \mathrm{MeV})$ like in a preequilibrium emission. It is not so surprising for this emission which reflects memory of the projectile velocity, that intrinsic rate is shorter than the time required to arrive at the scission point.

Let us quote the main conclusion given by Alexander et al. : "Even prior to the very rapid fission and quasi-fission processes, "quasi-fission" and "quasievaporation" must be taking place. These processes as well as their forward-peaked preequilibrium cousins provide a mean of studying the reaction process on a time scale comparable to that for energy damping in the entrance channe1."

Moreover, low temperature ${ }^{4} \mathrm{He}$ emission seems to occur from composite nuclei with angular momenta between 0 and $135 \hbar$ while the higher temperature emission comes from even higher spin states.

Very recently, forward linear momentum transferred to the target nucleus were measured $\left({ }^{87}\right)$ in the reaction $\left({ }^{16} 0+{ }^{238} \mathrm{U}\right)$ at $20 \mathrm{MeV}$ per nucleon. A kinematical analysis of the coincidences between projectiles residues ( $N, C, B, B e$ ) and fission fragments resulting from the target like residues has shown that the mechanism of momentum transfer and energy dissipation at such a rather high velocity is closely connected with the fast emission of a "jet" of particles emitted preferentially into the forward direction.

\subsection{Fragment de-excitation by sequential fission}

When the heaviest fragment in the two-body break-up has a $Z^{2} / A$ value high enough and shares an angular momentum, it is recognized that it undergoes fission. It was already noticed in the discovery of quasi-fission $\left({ }^{27}\right)$ for the system $\left({ }^{84} \mathrm{Kr}+{ }^{238} \mathrm{U}\right)$, that a large part of the heavy partner contribution was not observed at the expected correlation angle, and the hypothesis of sequential fission for Uranium like excited fragments was made $\left({ }^{8 B}\right)$. A detailed illustration of the 
method used to estimate the fraction of missing coincidences on the heavy fragment side is shown in figure 40 due to Rajagopalan et al $\left({ }^{89}\right)$. In this study of sequential fission of $\left({ }^{86} \mathrm{Kr}+{ }^{197} \mathrm{Au}\right)$ at $730 \mathrm{MeV}$ the authors have demonstrated that the Au-like products emerge from the deep inelastic collision with intrinsic spins comparable to ${ }^{194} \mathrm{Hg}$ compound nuclei which were studied $\left({ }^{84}\right)$ separatly by bombardment of ${ }^{182} \mathrm{~W}$ by ${ }^{12} \mathrm{C}$. Since in the last case the fission fraction exceeds 0.5 at $\mathrm{J}>31 \mathrm{~h}$ for $\mathrm{E}^{*}=98 \mathrm{MeV}$, typical spins in the range $30-40 \hbar$ are estimated for $Q=200 \mathrm{MeV}\left(E^{*}=140 \mathrm{MeV}\right)$ for the Au-like products from the deep inelastic collisions.

The sequential fission study and particularly the out of plane angular distribution has been used in order to extract the intrinsic angular momentum of the heavy fragment. These studies have been initiated by Vandenbosch et al $\left({ }^{90}\right)$. They assumed that the kinetic energy loss has been transferred to intrinsic excitation energy and rotational energy and that the statistical theory could be
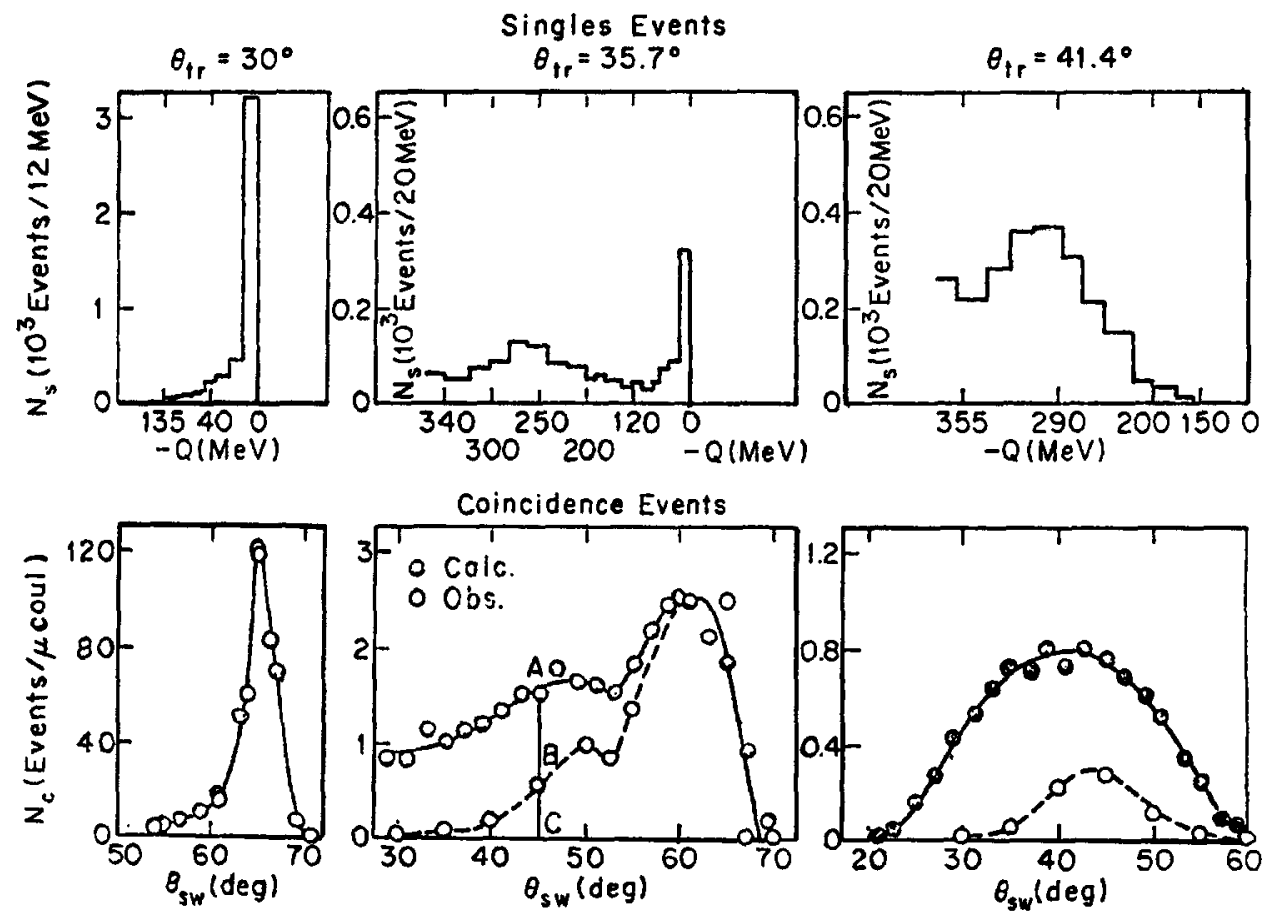

Fig. 40 : (top) Energy loss spectra observed at three angles for the system $\left({ }^{86} \mathrm{Kr}+197 \mathrm{Au}\right)$ at $730 \mathrm{MeV}$.

(bottom) Comparison of calculated and observed angular correlations for coincidence events between $\mathrm{Kr}-1 i k e$ and Au-like fragments. The fraction of missing coincidences at each sweep angle is the ratio ( $A B / B C)$. The $Q$ scales in the upper parts correspond with the $Q_{s w} s c a l e$ in the lower part. The data have been obtained at three trigger angles : $30^{\circ}, 35.7^{\circ}$ and $41.4^{\circ}$. At $30^{\circ}$ the predominant component of $\mathrm{Kr}-$ like fragments is quasi-elastic. At $41.4^{\circ}$, all observed events are from very deep inelastic collisions. The "sweep" detector was moved through the angular range $\theta_{\text {Sw }}=30^{\circ}-70^{\circ}$ to measure the angular correlations of the heavy fragment (ref. 89) 
applied to the fragments sharing the excitation energy and the spin. A nice example of the correlation between the kinetic energy loss and the fission probability is given by Specht $\left({ }^{91}\right)$ for the four systems $\left({ }^{58} \mathrm{Ni}+{ }^{20 \mathrm{Bbb}}\right),\left({ }^{10} \mathrm{Zr}+{ }^{208 \mathrm{~Pb}}\right)$ and $\left({ }^{58} \mathrm{Ni}+{ }^{238} \mathrm{U}\right)\left({ }^{90} \mathrm{Zr}+238 \mathrm{U}\right)$.

At the present stage, we may conclude that most of the data are in favour of a nearly complete transfer of the kinetic energy loss into intrinsic excitation of the fragments. However we have shown that in some cases, peculiar effects appear which, even if they correspond to a small fraction of the total kinetic energy loss, can give useful informations on the mechanism of the energy dissipation. It might be that a well choosen set of light particles emitted with very typical characteristics reflect important aspects of the mechanism of energy damping. Several theoretical models have appeared - Fermi jets, single fast particle emission following giant resonance absorption, "piston" effect - but none of them has been really observed experimentally.

As a conclusion, we may surmarize our knowledge on the dissipation of kinetic energy in heavy ion collisions. At the contact time, which lasts less than $10^{-22} \mathrm{sec}$, the coherent motion of nucleons is a diabatic process during which the first stage of energy damping occurs. There are some indications that when the energy attains around $8 \mathrm{MeV}$ per nucleon a few light particles are emitted at that stage. Surface vibrations are also quite likely and they may be disclosed by the observation of structures in fragment energy spectra corresponding to giant resonances.

Between $10^{-22}$ and $10^{-21}$, the highly correlated motion decays into many states (collective excitation, particle-hole excitation, nucleon exchange). The neutron to proton ratio undergoes an important change from the projectile and target ratio to an equilibrium value which is determined by the minimalization of the composite system potential energy. This is probably governed by isovector dipole giant resonance, but the statistical aspects of the phenomena is also occuring. While there are veryclear-cut results on the $N / Z$ equilibration, it is not so well proved that they are due to the giant resonance process.

The third stage is the well explored domain of statistical aspects, for which the transport theories have been so successful. The heat bath has been created by the two first steps and the kinetic energy loss increases when time evolves between $10^{-21}$ and $10^{-22} \mathrm{sec}$. Mass and charge distributions are broadening during this period but the energy loss is achieved (complete relaxation) in the quasi-fission events, before the end of the mass spreading, since the mass distribution width increases during $10^{-20} \mathrm{sec}$. The angular momentum transfer, as disclosed by gamma multiplicity, sequential fission and particle emission measurements, follows the radial kinetic energy loss, so that a tangential component has to be added.

A composite system has been formed which rotates for a while, the angle of rotation depending mainly of $n^{\prime}=z_{1} z_{2} e^{2 / \pi v^{\prime}}$. Whether such a system corresponds or not to classical sticking is a matter of asymmetry and total mass inertia. Before to disrupt into two fragments, the system is certainly deformed as this is clearly shown by the magnitude of the totally relaxed mean kinetic energy of the fragments, $\langle E\rangle$. The width of this quasi-fission kinetic energy is also an interesting data for the knowledge of the shape of the system, and of the neck behaviour. There are also some indications that a few light particles might be emitted, in some cases, from the neck.

The study of the de-excitation of the fragments is another interesting piece of information. From most of the available data, and more particularly from neutron emission extensive studies, it appears that the nuclear temperature is the 
same in the light and in the heavy fragment. The final excitation energy, shared by the fragments just before particle emission is strictly proportionnal to the number of nucleons. This means that the deformation energy has not been greater in the light than in the heavy fragment, or more precisely that it is also proportionnal to the volume (or to $A$ ) and not to the surface $\left(A^{2} / 3\right.$ ) or independent of $A$, as this could be if collective surface vibration modes would have a leading role in the final stage of energy dissipation.

\section{ACKNOWLEDGMENTS}

Most of the material shown in this survey has been taken from the results obtained at Berkeley, Darmstadt and Orsay. I should like to thank all the authors from the Lawrence Berkeley Laboratory, from Rochester University, Washington State University in U.S.A., from M.P.I. Heidelberg and G.S.I. Darmstadt in Germany, from Institute of Modern Physics in Lanchow in China and from Institut de Physique Nucléaire Orsay, who have provided published data, preprints and unpublished results. I am particularly indebted to Prof. J.M. Alexander from New York State University (Stony Brook) for illuminating discussions during his visit in Orsay.

\section{REEERENCES}

( $\left.{ }^{1}\right)$ A. Fleury, J.M. Alexander, Ann. Rev. Nucl. Sci. 24, 279 (1974).

$\left({ }^{2}\right)$ J. Galin, J. de Physique 37, C.5. 83 (1976).

$\left({ }^{3}\right)$ L. Moretto, R.P. Schmitt, J. de Physique 37, C.5 109 (1976).

$\left({ }^{4}\right)$ M. Lefort, Reports Prog. in Phys. 39, 129 (1976).

$\left({ }^{5}\right)$ W.V. Schröeder, S. Hui zenga, Ann. Rev. Nucl. Sci. 27, 465 (1977).

$\left({ }^{6}\right)$ M. Lefort, C. Ngô, Annales Physique $\underline{3}, 5-114$ (1978).

${ }^{7}$ ) V.V. Volkov, Physics Reports, 44C, 93 (1978).

$\left({ }^{8}\right)$ D.H.E. Gross, H. Kalinowski, Physics Reports 45, 176 (1978).

$\left({ }^{9}\right)$ R. Babinet, B. Cauvin, J. Girard, H. Nifenecker, B. Gatty, D. Guerreau, M. Lefort, X. Tarrago, Nuc7. Phys. A296, 160 (1978).

$\left({ }^{10}\right)$ A. Cauvin, R.C. Jared, P. Russo, R.P. Schmitt, R. Babinet, L. Moretto, Nucl. Phys. A301, 511 (1977).

(11) J. Péter, M. Berlanger, C. Ngô, B. Tamain, B. Lucas, C. Mazur, M. Ribrag, C. Signarbieux, Z. Phys. A238, 413 (1977).

(12) Y. Eyal, A. Gavron, I. Tserruya, Z. Fraenke1, Y. Eisen, S. Wald, R. Bass, C.R. Gould, G. Kreislind, R. Renfort, K. Stelzer, R. Zitsmann, A. Gobbi, U. Lynen, I. Rode, Phys. Rev. Lett. 41, 625 (1978).

$\left({ }^{13}\right)$ R. Kaufmann, R. Wolfgang, Phys. Rev. 121, 192 (1961).

(14) J. Galin, B. Gatty, M. Lefort, J. Péter, X. Tarrago, R. Basile, Phys. Rev. 182, 1267 (1969) ; Nuc7. Phys. A159, 461 (1970).

$\left({ }^{15}\right)$ J. Galin, D. Guerreau, M. Lefort, X. Tarrago, J. de Physique Paris, 32, 7 (1971).

$\left({ }^{16}\right)$ G.F. Gridnev, V.V. Volkov, J. Wilczynski, Nucl. Phys. A142, 385 (1970).

$\left({ }^{17}\right)$ a- A.G. Artukh, A.G. Avdeichikov, J. Ero, G.F. Grichev, V.L. Mikheev,

V.V. Volkov, J. Wilczynski, Nucl. Phys. A160, 511 (1971) ; A168, 321 (1971)

b- A.G. Artukh, G.F. Gridnev, V.L. Mikheev, V.V. Volkov, J. Wilczynski, Nucl. Phys. A211, 299 (1973) ; A215, 91 (1973). 
$\left({ }^{18}\right)$ J. Wilczynski, Phys. Lett. $\underline{47 B}, 484$ (1973).

$\left({ }^{19}\right)$ Wu Zhong Li, Chu Yung Tai, Xia Guo Zhong, Liu Bu Sheng, Li Fa Wei, Fan Guo Ying, Institute of Modern Physics, Preprint LANCHOW China (1978).

$\left({ }^{20}\right)$ B. Gatty, D. Guerreau, M. Lefort, X. Tarrago, J. Galin, B. Cauvin, J. Girard, H. Ni fenecker, Nucl. Phys. A253, 511 (1975).

$\left({ }^{21}\right)$ W.U. Schroëder, J.R. Birkelund, J.R. Huizenga, K.L. Wolf, V.E. Viola Jr, Phys. Reports 45, 302 (1978).

${ }^{22}$ ) S. Agarwal, J. Galin, B. Gatty, D. Guerreau, M. Lefort, X. Tarrago, R. Babinet, B. Cauvin, J. Girard, H. Ni fenecker, Nucl. Phys. A293, 230 (1977).

$\left({ }^{23}\right)$ J. Pêter, C. Ngô, F. Plasil, B. Tamain, M. Berlanger, F. Hanappe, Nucl. Phys . A279, 110 (1977).

$\left({ }^{24}\right)$ J. Galin, L.G. Moretto, R. Babinet, R. Schmitt, R. Jared, S.G. Thompson, Nuc]. Phys. A255, 472 (1975).

$\left({ }^{25}\right)$ L.G. Moretto, J. Galin, R. Babinet, Z. Fraenke 1, R. Schmitt, R. Jared, S.G. Thompson, Nucl. Phys. A259, 173 (1976).

$\left({ }^{26}\right)$ C. Ngô, J. Péter, B. Tamain, M. Berlanger, F. Hanappe, Z. Phys. A283, 161 (1977).

$\left({ }^{27}\right)$ F. Hanappe, M. Lefort, C. Ngô, J. Péter, B. Tamain, Phys. Rev. Lett. 32 , 738 (1974).

$\left({ }^{28}\right)$ R. Babinet, L.G. Moretto, J. Galin, R. Jared, J. Moulton, S.G. Thompson, Nucl. Phys. A258, 172 (1976).

$\left({ }^{29}\right)$ J.C. Jacmart, P. Colombani, H. Doubre, N. Frascaria, N. Poffe, M. Riou, J.C. Roynette, C. Stéphan, A. Weidinger, Nucl. Phys. A242, 175 (1975).

$\left({ }^{30}\right)$ K.L. Wolf, C.T. Rodre, Proc. Symp. Macroscopic Features Heavy Ion Collisions Report ANL/Phys. 76.2. Vol. II (1976).

$\left({ }^{31}\right)$ S. Agarwal, Thesis Dr of Sciences, Orsay (1979).

$\left({ }^{32}\right)$ R. Vandenbosch, M.P. Webb, P. Dyer, R.J. Puigh, T.D. Thomas, M.S. Zisman, Preprint (1978).

$\left({ }^{33}\right)$ G. Rudolf, A. Gobbi, H. Stelzer, R. Bock, H. Sann, U. Lynen, A. Olmi, D. Pelte, Jahresbericht, G.S.I. J.1.78, 41 (1978).

$\left({ }^{34}\right)$ G. Rudolf, A. Gobbi, H. Stelzer, U. Lynen, A. 01mi, H. Sann, R.G. Stokstad, D. Pelte, G.S.I. VP.12.78 (1978).

$\left({ }^{35}\right)$ J.R. Huizenga, J.R. Birkelund, W.U. Schröder, K.L. Wolf, V.E. Viola, Phys . Rev. Lett. 37, 885 (1976).

$\left({ }^{36}\right)$ T. Cormier, P. Braun-Munzinger, P.M. Cormier, J.W. Harris, L.L. Lees, Phys . Rev. C16, 215 (1977).

$\left({ }^{37}\right)$ J.B. Natowitz, G. Doukellis, B. Kolb, G. Rosner, Th. Walcher, Max Planck Insti tut Report, V18 (1978).

$\left({ }^{38}\right)$ P.J. Siemens, J.P. Bondorf, D.H.E. Gross, F. Kickmann, Phys . Lett. 360,24 (1971).

$\left({ }^{39}\right)$ W. Schroëder, J.R. Huizenga, J.B. Birkelund, K.L. Wolf, V.E. Viola, Phys. Lett. 71B, 283 (1977).

$\left({ }^{40}\right)$ J.R. Huizenga, W.U. Schroëder, J.R. Birkelund, W. Wilcke, Fall Creek Falls Park meeting (June 1977) UR.NSRL.152 (1977).

( ${ }^{4}$ ) J. Blocki, J. Randrup, W.J. Swiatecki, C.F. Tsang, Ann. Phys. 105, 427 (1977). J. Randrup, Ann. Phys. 112, 356 (1978).

J. Randrup, Fali Creek FaTis, Oak Ridge, Conf. 770602, 218 (1977). 
$\left({ }^{42}\right)$ S.E. Koonin, R.L. Hatch, J. Randrup, Nucl. Phys. A277, 509 (1977).

J. Randrup, J.S. Vaagen, Preprint Nordita 78/9.

$\left({ }^{43}\right)$ G. Rudolf, A. Gobbi, H. Stelzer, U. Lynen, A. $01 \mathrm{mi}$, H. Sann, R. Stokstad, D. Pelte, Preprint (1978).

$\left({ }^{44}\right)$ D.H.E. Gross, H. Kalinowski, J.N. De, Symposium classical and quantum mechanical aspects of heavy ion collisions, Heidelberg 1974, Lecture notes in Physics Springer Verlag, 33, 194 (1975).

$\left({ }^{45}\right)$ H.H. Deubler, K. Dietrich, Phys. Lett. B56, 241 (1975).

$\left({ }^{46}\right)$ W.U. Schroëder, J.R. Birkelund, J.R. Huizenga, K.L. Wolf, V.E. Viola Jr, Phys. Rev. C16, 623 (1977).

$\left({ }^{47}\right)$ G. Wolschin, N. Norenberg, Z. Phys. A284, 216 (1978).

W. Nörenberg, Z. Phys. A274, 841 (1975).

$\left({ }^{48}\right)$ S. Ayik, B. Schürmann, W. Nörenberg, Z. Phys. A279, 145 (1976).

(49) C. Riede 1, G. Wolschin, W. Nörenberg, G.S.I. VT.10.78 (1978).

( $\left.{ }^{50}\right)$ M. Aleonard, G.J. Wozniak, P. Glässe 1, M.A. Deleplanque, R.M. Diamond, L.G. Moretto, R.P. Schmitt, F.S. Stephens, Phys. Rev. Lett. 40,622 (1978).

$\left({ }^{51}\right)$ W. Nörenberg, Phys. Lett. 53B, 289 (1974).

$\left({ }^{52}\right)$ See for example W. Schroëder and J.R. Huizenga, ref. 5, p. 531.

$\left({ }^{53}\right)$ R.A. Broglia, C.H. Dasso, A. Winther, Phys. Lett. 61B, 113 (1976).

$\left({ }^{54}\right)$ R.R. Betts, S.B. Dicenzo, M.H. Mortensen, R.L. White, Phys. Rev. Lett. 39 , 1183 (1977).

$\left({ }^{55}\right)$ M. Buenerd, D. Lebrun, J. Chauvin, Y. Gaillard, J.M. Loiseaux, P. Martin, G. Perrin, P. de Saintignon, Phys. Rev. Lett. 40, 1482 (1978).

$\left({ }^{56}\right)$ P. Doll, D.L. Hendrie, J. Mahoney, A. Menchaca-Rocha, D.K. Scott, T.J.M. Symons, K. van Bibber, Y.P. Viyogi, H. Wieman, Phys. Rev. Lett. 42 , $366(1979)$.

$\left({ }^{57}\right)$ N. Frascaria, L. Tassan-Got, P. Colombani, J.P. Garron, J.C. Jacmart, M. Riou, C. Stéphan, Phys. Rev. Lett. 39, 198 (1977).

$\left({ }^{58}\right)$ N. Frascaria, 5 ème session d'études biennales de physique nucléaires, Aussois (1979), IPNO.PhN.79.04.

$\left({ }^{59}\right)$ R.A. Broglia, C.H. Dasso, G. Pollarolo, A. Winther, Phys. Rev. Lett. 41,25 (1978).

$\left({ }^{60}\right)$ M. Goldhaber, E. Teller, Phys. Rev. 74, 1046 (1948).

$\left({ }^{6}{ }^{1}\right)$ M. Berlanger, A. Gobbi, F. Hanappe, U. Lynen, C. Ngô, A. O1mi, H. Sann, H. Stelzer, H. Richel, M.F. Rivet, Hirschegg winter meeting (1979) and Z. Phys. (1979).

$\left({ }^{62}\right)$ B. Gatty, D. Guerreau, M. Lefort, J. Pouthas, X. Tarrago, J. Galin, B. Cauvin, H. Ni fenecker, Nucl. Phys. A278, 347 (1976).

$\left({ }^{6}{ }^{3}\right)$ L.G. Moretto, J. Sventek, G. Mantzouranis, Phys. Rev. Lett. 42, 563 (1979).

$\left({ }^{64}\right)$ P. Auger, T.H. Chiang, J. Galin, B. Gatty, J. Girard, D. Guerreau, E. Nolte, J. Pouthas, X. Tarrago, Winter meeting Hirschegg (1979).

$\left({ }^{65}\right)$ W. Nörenberg, J. Phys. 37 C 5,141 (1976).

$\left({ }^{66}\right)$ R.P. Schmitt, G. Bizard, G.J. Wozniak, L.G. Moretto, Phys. Rev. Lett. 41 , 1152 (1978).

$\left({ }^{67}\right)$ C.K. Gelbke, P. Braun-Munzinger, J. Barette, B. Zeidman, M.J. Levine, A. Gamp, H.L. Harney, Th. Walcher, Nucl. Phys. A269, 460 (1976). 
$\left({ }^{6}{ }^{8}\right)$ R. Babinet, B. Cauvin, J. Girard, H. Nifenecker, B. Gatty, D. Guerreau, M: Le fort, X. Tarrago, Nucl. Phys. A296, 60 (1978).

$\left.{ }^{69}\right)$ B. Cauvin, R.C. Jared, P. Russo, R.P. Schmitt, R. Babinet, L.G. Moretto, Nucl. Phys. A301, 511 (1978).

$\left({ }^{70}\right)$ J.C. Roynette, H. Doubre, N. Frascaria, J.C. Jacmart, N. Poffe, M. Riou, Phys. Lett. 678, 395 (1977).

$\left({ }^{71}\right)$ J. Péter, M. Berlanger, C. Ngõo, B. Tamain, B. Lucas, C. Mazur, M. Ribrag, C. Signarbieux, Z. Phys. A283, 413 (1977).

$\left.{ }^{72}\right)$ B. Tamain, R. Chechik, H. Fuchs, F. Hanappe, M. Morjean, C. Ngô, J. Péter, M. Dakowski, B. Lucas, C. Mazur, M. Ribrag, C. Signarbieux, Nuci. Phys. (in press).

$\left({ }^{73}\right)$ C.R. Gould, R. Bass, J.R. Czarnecki, V. Hartmann, K. Stelzer, R. Zitzmann, Y. Eyal, Z. Phys. A284, 353 (1978).

$\left({ }^{74}\right)$ Y. Eyal, A. Gavron, I. Tserruya, Z. Fraenke 1, Y. Essen, S. Wald, R. Bass, C.R. Gould, G. Kreyling, R. Renfordt, K. Stelzer, R. Zitzamnn, A. Gobbi, U. Lynen, H. Stelzer, I. Rode, R. Bock, Phys. Rev. Lett. 41, 625 (1978).

$\left({ }^{75}\right)$ S. Agarwal, R. Babinet, B. Cauvin, J. Girard, J. Alexander, P. Auger, T.H. Chiang, J. Galin, B. Gatty, D. Guerreau, X. Tarrago, to be published.

$\left({ }^{76}\right)$ T. Ericson, V. Strutinski, Nucl. Phys. 8,284 (1958).

( $\left.{ }^{77}\right)$ C.K. Gelbke, M. Bini, C. Olmer, D.L. Hendrie, J.L. Laville, J. Mahomey, M.C. Mermaz, D.K. Scott, H.H. Wieman, Phys. Lett. 71B, 83 (1977).

$\left({ }^{78}\right)$ T. Inamura, M. Ishihara, T. Fukuda, T. Shimoda, K. Hiruta, Phys . Lett. 68B, 51 (1977).

$\left({ }^{79}\right)$ T. Schimoda, M. Ishihara, H. Kamitsubo, T. Motobayashi, T. Fukuda, IPCR (1977).

$\left({ }^{80}\right)$ J.W. Harris, T.M. Cormier, D.F. Geesaman, L.L. Lee, R.L. Mac Grath, J.P. Wurm, Phys. Rev. Lett. 38, 1460 (1977).

$\left({ }^{81}\right)$ H. Ho, R. Albrecht, W. Dunnweber, G. Graw, S.G. Steadman, J.P. Wurm, D. Disdier, V. Rauch, F. Scheibling, Z. Phys. A283, 235 (1977).

( $\left.{ }^{82}\right)$ M. Blann, Phys. Rev. Lett. 21,1375 (1966).

( $\left.{ }^{83}\right)$ J.M. Miller, G.L. Catchen, D. Logan, M. Rajagopalan, J.M. Alexander, M. Kaplan, M.S. Zisman, Phys. Rev. Lett. 40, 100 (1978).

$\left({ }^{84}\right)$ J.M. Miller, D. Logan, G.L. Catchen, M. Rajagopalan, J.M. Alexander, M. Kaplan, J.W. Bal1, M.S. Zisman, L. Kowalski, Phys. Rev. Lett. 40, 1074 (1978).

( ${ }^{85}$ ) A. Gamp, J.C. Jacmart, N. Poffé, H. Doubre, J.C. Roynette, J. Wilczynski, Phys. Lett. 74B, 215 (1978).

$\left({ }^{86}\right)$ D. Logan, M. Rajagopalan, M.S. Zisman, J.M. Alexander, M. Kaplan, L. Kowalski, Preprint (1979).

$\left({ }^{87}\right)$ P. Dyer, T.C. Awas, C.K. Gelbke, B.B. Back, A. Mignery, K.L. Wolf, H. Brener, V.E. Viola Jr, W.G. Meyer, Phys. Rev. Lett. 42, 560 (1979).

$\left.{ }^{88}\right)$ B. Tamain, Thesis, Clermont-Ferrand (1974) $n^{\circ} 182$.

M. Lefort, J. Péter, C. Ngô, B. Tamain, Nuc1. Phys. A216, 166 (1973).

( $\left.{ }^{89}\right)$ M. Rajagopalan, L. Kowalski, D. Logan, M. Kaplan, J.M. Alexander, M.S. Zisman, J.M. Mi 1ler, Phys. Rev. C19, 54 (1979).

(90) P. Dyer, R.J. Puigh, R. Vandenbosch, T.D. Thomas, M.S. Zisman, Phys. Rev. Lett. 39, 392 (1977).

$\left({ }^{91}\right)$ H.J. Specht, M.P.I. Report H.V. 36 (1978). 
$\left({ }^{92}\right)$ M. Berlanger, R. Chechik, H. Fuchs, F. Hanappe, M. Morjean, J. Pēter, B. Tamain, M. Dakowski, B. Lucas, C. Mazur, M. Ribrag, C. Signarbieux, Int. Conf. Physics and Chemistry of Fission, IAEA, Julich, May 1979, SM 241, p. 132

$\left({ }^{93}\right)$ K. Hartmann, personnal communication, Munich University.

$\left({ }^{94}\right)$ D. Hischer, J.R. Birkelund, A.D. Hoover, W.U. Schröder, W.W. Wilcke, J.R. Huizenga, A. Mi gnerey, K. Wolf, H.F. Brener, V.E. Viola Jr, Preprint U.R. NSRL.189 (1979). 